U. S. DEPARTMENT OF COMMERCE

JESSE H. JONES, Secretary

NATIONAL BUREAU OF STANDARDS

LYMAN J. BRIGGS, Director

\title{
STANDARD STOCK PONDEROSA PINE DOORS
}

\section{COMMERCIAL STANDARD CS120-44}

Effective Date for New Production from September 15, 1944

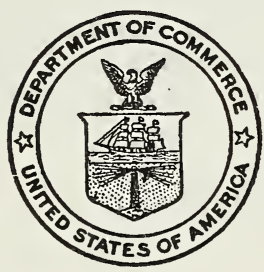

\section{A RECORDED VOLUNTARY STANDARD OF THE TRADE}

UNITED STATES

GOVERNMENT PRINTING OFFICE

WASHINGTON : 1944 


\section{P R O M U L G A T I N}

of

COMMERCIAL STANDARD CS120-44

for

\section{STANDARD STOCK PONDEROSA PINE DOORS}

On January 29, 1944, at the instance of the National Door Manufacturers Association, a proposed commercial standard for standard stock ponderosa pine doors was submitted to manufacturers, testing laboratories, and distributor and consumer organizations for comment: An adjusted draft was then circulated to the trade for written acceptance. Those concerned have since accepted and approved the standard as shown herein for promulgation by the United States Department of Commerce, through the National Bureau of Standards.

This standard is effective for new production from September 15, 1944.

Promulgation recommended.

I. J. Fairchild,

Promulgated.

Chief, Division of Trade Standards.

Lyman J. Briggs,

Promulgation approved. Director, National Bureau of Standards.

Jesse H. Jones, Secretary of Commerce. 


\section{STANDARD STOCK PONDEROSA PINE DOORS ${ }^{1}$}

\section{COMMERCIAL STANDARD CS120-44}

\section{PURPOSE}

1. The purpose of this commercial standard is to establish standard specifications and sizes for ponderosa pine, standard stock doors to guide producers, distributors, architects, builders, and the public; to provide a uniform basis for guaranteeing compliance through the use of labels or certifications; to avoid delays and misunderstandings; and to effect economies from the producer to the ultimate user through a wider utilization of standard, ponderosa pine doors.

2. In the development of this standard every effort has been made to include designs which will permit freedom of architectural expression. Ponderosa pine doors will continue to be available for all types of architectural designing.

3. To meet the modern trend toward economy and simplification of installation, doors may be specified "Prefit" to the exact size required. (See par 11.) Doors will be mortised for locks and cut for hinges when so specified.

\section{SCOPE}

4. This standard provides minimum specifications for stock ponderosa pine doors in four nominal thicknesses, $3 / 4,1 \frac{1}{8}, 1 \frac{3}{8}$, and $1 \frac{3}{4}$ inches. It covers construction, grades, and tolerances for these requirements.

4a. There are standard stock lay-outs and designs for the following:

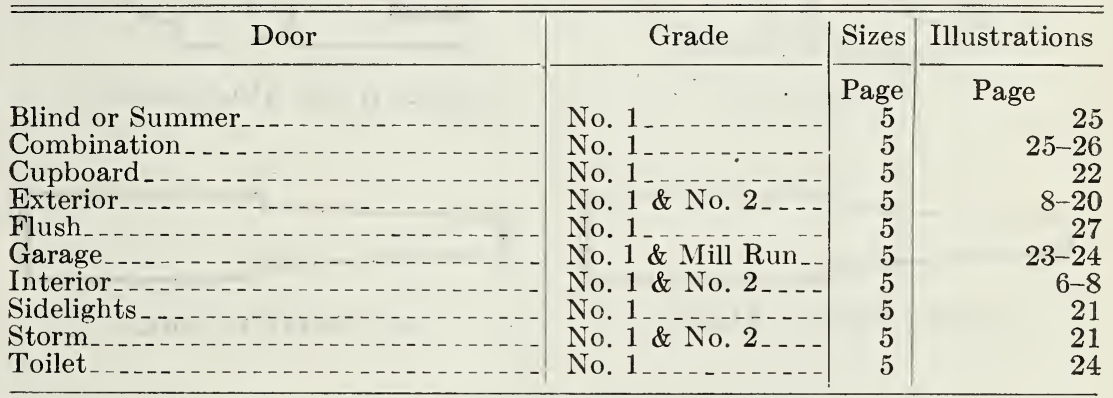

\section{GENERAL REQUIREMENTS}

5. All commercial standard ponderosa pine panel and sash doors shall meet the following requirements:

6. Material.-Doors shall be made of properly kiln-dried ponderosa pine.

7. Workmanship.-Doors shall be well manufactured and machined, with flat faces of stiles, rails, and panels smoothly machine sanded.

8. Construction.- Panel and sash doors shall be assembled by what is known as "dowelled construction," that is, stiles and rails to be bored to receive dowels not less than $3 / 8$-inch in diameter by approximately

1 Ponderosa pine, one of the Western pines, has proved over the past 40 years to be highly adaptable for woodwork. This pine is light in color, ranging from creamy-white to straw color. The grain is close, uniform, and resists raising. The surface is even-textured. It takes nails and screws without splitting, is easy to mortise for locks and cut for hinges. It sands to a satin-smooth finish, takes paint, enamel, stain, and varnish, holding them well. The ends and edges do not splinter easily. 
$4 \frac{3 / 4}{4}$ inches long for doors $3 / 4$-inch thick, and not less than $1 / 2$-inch in diameter by approximately 5 inches long for doors $1 \frac{1 / 8}{8}, 1 \frac{3 \pi}{8}$, and $1 \frac{3}{4}$ inches thick. (Except that cupboard doors and narrow stile doors may have shorter dowels.) Dowels shall have glued grooves and be

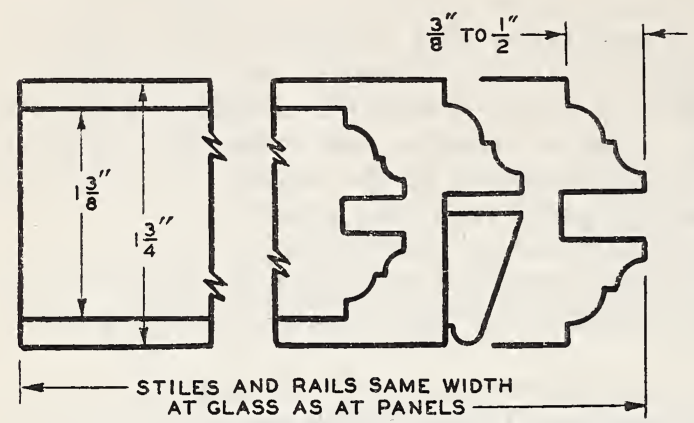

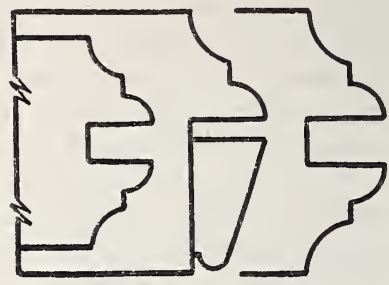

COVE AND BEAD

BEAD AND COVE

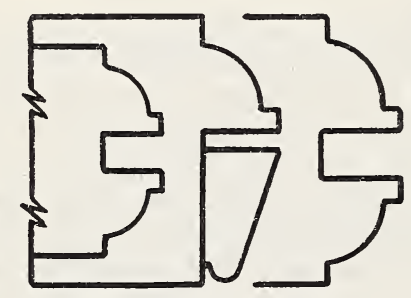

OVOLO "A"

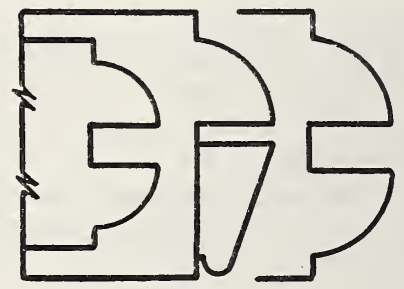

OVOLO "B" OR RULE JOINT

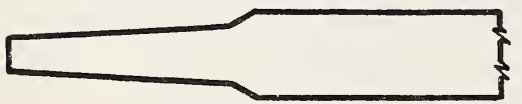

BEVEL RAISED PANEL

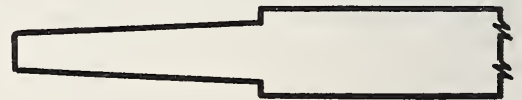

HIP RAISED PANEL

\section{FLAT PLYWOOD PANEL}

Figure 1.-Sticking and panel details.

to a drive fit. Dowels shall be set in water-resistant glue and shall extend approximately one-half their length into each stile and rail, and assembled under pressure. Because of the fact that all present standard door-boring machines are built for $2 \frac{1 / 4}{4}$-inch dowel centers, the quantity of dowels used is limited according to the width of the rails and shall be based on the following minimum number of dowels at each end of rails:

Rails under 41/4 inches wide ............ 1 dowel.

Rails $4 \frac{1}{4}$ inches to 7 inches wide....... 2 dowels.

Rails over 7 inches wide............. 3 dowels, plus one additional dowel for each additional full 3 inches in width. 
8a. At the option of the manufacturer, doors may be assembled by what is known as blind-mortised and tenoned construction instead of dowelled construction.

9. Sticking.-Stiles and rails shall have solid sticking. All intersections shall be coped with joints well-fitted. "Cove and Bead," "Bead and Cove," "Ovolo A" or "Ovolo B or Rule Joint" sticking shall be standard on all standard ponderosa pine doors. See figure 1 . Imperfect sticking which may develop in machining shall be carefully repaired or neatly replaced. Panels are also illustrated in figure 1.

10. Thicknesses.-Doors shall be of the following thicknesses; and a thickness tolerance of minus $1 / 16$ inch shall be allowed:

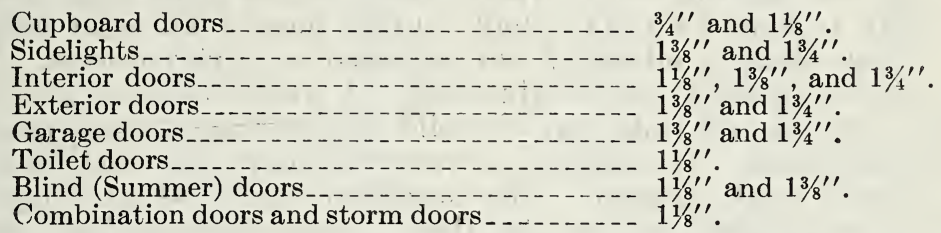

11. Size tolerance:- Unless otherwise specified, a height and width tolerance of plus $1 / 8$ inch shall be allowed. When ordered "Prefit", doors will be made to standard opening widths and heights with a tolerance of plus or minus $1 \frac{1}{32}$ inch. "Prefit" doors shall have skid blocks, strips, or other type of protector.

\section{GRADING}

12. All doors shall be graded according to both sides or faces. A shipment of any grade shall represent a fair average of that grade.

13. Ordinarily, interior, exterior, and storm doors can be obtained in grades "No. 1" and "No. 2"; cupboard doors, sidelights, casement doors, toilet doors, blind doors, combination doors, and flush doors in grade "No. 1" only; and garage doors in grades "No. 1" and "Mill Run."

\section{GRADE “NO. 1"--Recommended for Natural, Stain, or Paint Finish}

14. Stiles and rails. - This stock shall be practically clear. Bright sap, light-brown stain, and light-red kiln burn shall be permitted. Each stile or bottom rail may contain one carefully repaired pitch seam on each side, provided it does not extend through the piece nor exceed $2 \frac{1}{2}$ inches in length. Rails wider than $4 \frac{3}{4}$ inches may be glued up with not over one joint up to $93 / 4$ inches, two joints up to 12 inches, and with not more than the same proportion of joints being permitted in wider rails. A water-resistant glue shall be used. Stiles and rails may be solid or veneered at the option of the manufacturer. If veneered, a water-resistant glue shall be used.

15. Panels-flat plywood.-The standard thickness of 3-ply flat plywood panels shall be not less than $1 / 4$ inch after sanding except inner frame and cupboard doors, which shall be not less than $3 / 16$ inch. If ponderosa pine, they shall be "sound and better two sides," according to standard commercial grading rules issued by pine plywood manufacturers; if fir, they shall be "sound two sides," according to Commercial Standard CS45-42; if hardwood, they shall conform to the generally accepted grades of door panels.

16. Panels - solid. - The standard thickness of solid ponderosa pine panels raised two sides shall be not less than $7 / 16$ inch after sand- 
ing, and shall conform to the grade of the stiles and rails. Panels wider than $93 / 4$ inches may be glued-up. A water-resistant glue shall be used.

GRADE "NO. 2".--Recommended Primarily for Paint Finish

17. Stiles and rails.-This stock may contain light blue stain, medium-brown stain, or medium-red kiln burn showing on not to exceed 50 percent of the area of any piece, as well as pitch streaks, checks, pitch pockets if carefully slivered, tight sound knots not to exceed $5 / 8$ inch in diameter, and other imperfections, not one of which shall be more serious in nature than those already enumerated. Each stile shall contain one such imperfection, but no piece shall contain more than two, and no door shall contain more than eight on each side. Plugs shall be admitted but regarded as imperfections. Rails wider than $4 \frac{3 / 4}{4}$ inches may be glued-up. A water-resistant glue shall be used. Stiles and rails may be solid or veneered at the option of the manufacturer. If veneered, a water-resistant glue shall be used.

18. Panels-flat plywood.-The standard thickness of 3-ply flat plywood panels shall be not less than $1 / 4$ inch after sanding, except inner frame and cupboard doors, which shall be not less than $3 / 16$ inch. If ponderosa pine, they shall be "sound and better two sides" according to standard commercial grading rules issued by pine plywood manufacturers; if fir, they shall be "sound two sides," according to Commercial Standard CS45-42.

19. Panels-solid.-The standard thickness of solid ponderosa pine panels raised two sides shall be not less than $7 / 16$ inch after sanding and shall conform to the grade of the stiles and rails. Panels wider than $9 \frac{3 / 4}{4}$ inches may be glued-up. A water-resistant glue shall be used.

GRADE "MILL RUN".--Recommended for Paint Finish Only (Garage Doors Only)

20. "Mill Run" grade may contain blue stain, brown stain or redkiln burn, worm holes, checks, pitch streaks, pitch pockets, fine shake, tight sound knots not to exceed 2 inches in diameter, and other imperfections, none of which shall be more serious in nature than those already enumerated.

21. Panels-flat plywood.-The standard thickness of 3-ply flat plywood panels shall be not less than $1 / 4$ inch after sanding. If ponderosa pine, they shall be "sound and better two sides" according to standard commercial grading rules issued by pine plywood manufacturers; if fir, they shall be "sound two sides," according to Commercial Standard CS45-42.

22. Panels - solid.-The standard thickness of solid ponderosa pine panels raised two sides shall be not less than $7 / 16$ inch after sanding and shall conform to the grade of the stiles and rails. Panels wider than $9 \frac{3 / 4}{4}$ inches may be glued-up. A water-resistant glue shall be used.

\section{DESIGNS AND LAY-OUTS}

23. Measurements for stiles, rails, mullions, and muntins shown in lay-outs are over-all (face measurement plus the sticking). A tolerance of $1 / 8$ inch in width shall be permitted. Unless otherwise specified, glass measurements may vary not more than $1 / 4$ inch from those shown in the lay-outs. (These tolerances allow for variations in different manufacturers' practices.) 
24. Interior doors of any design narrower than 1 foot 6 inches in width will be furnished with stiles $3 \frac{3 / 4}{4}$ inches over-all width, unless otherwise specified.

TABLE 1.-Sizes

\begin{tabular}{|c|c|c|c|c|c|}
\hline \multicolumn{3}{|c|}{ Standard sizes, cupboard doors } & \multicolumn{2}{|c|}{$\begin{array}{l}\text { Standard sizes, interior and } \\
\text { exterior panel, sash, and } \\
\text { flush doors }\end{array}$} & $\begin{array}{l}\begin{array}{l}\text { Standard } \\
\text { sizes, } \\
\text { sidelights }\end{array} \\
1^{\prime} 0^{\prime \prime} \times 6^{\prime} 8^{\prime \prime} \\
1^{\prime} 2^{\prime \prime} \\
1^{\prime} 4^{\prime \prime} \\
1^{\prime} 6^{\prime \prime} \\
1^{\prime} 0^{\prime \prime} \times 6^{\prime} 10^{\prime \prime} \\
1^{\prime}{ }^{\prime \prime} \\
1^{\prime}{ }^{\prime \prime} \\
1^{\prime} 6^{\prime \prime}\end{array}$ \\
\hline $\begin{array}{l}1^{\prime} 0^{\prime \prime} \times 1^{\prime} 6^{\prime \prime} \\
1^{\prime} 2^{\prime \prime \prime} \\
1^{\prime} 4^{\prime \prime} \\
1^{\prime} 6^{\prime \prime} \\
1^{\prime} 8^{\prime \prime} \\
1^{\prime} 10^{\prime \prime} \\
2^{\prime} 0^{\prime \prime} \\
1^{\prime} 8^{\prime \prime} \times 11^{\prime \prime} 8^{\prime \prime} \\
1^{\prime} 10^{\prime \prime} \times 11^{\prime} 10^{\prime \prime} \\
1^{\prime} 0^{\prime \prime} \times 2^{\prime} 0^{\prime \prime} \\
1^{\prime} 2^{\prime \prime} \\
1^{\prime} 4^{\prime \prime} \\
1^{\prime} 6^{\prime \prime} \\
1^{\prime} 8^{\prime \prime} \\
1^{\prime} 10^{\prime \prime} \\
2^{\prime} 0^{\prime \prime} \\
1^{\prime} 0^{\prime \prime} \times 2^{\prime} 6^{\prime \prime} \\
1^{\prime} 2^{\prime \prime} \\
1^{\prime} 4^{\prime \prime} \\
1^{\prime} 6^{\prime \prime} \\
1^{\prime} 8^{\prime \prime} \\
1^{\prime} 10^{\prime \prime} \\
2^{\prime} 0^{\prime \prime} \\
1^{\prime} 0^{\prime \prime} \times 2^{\prime} 8^{\prime} \\
1^{\prime} 2^{\prime \prime} \\
1^{\prime} 4^{\prime \prime} \\
1^{\prime} 6^{\prime \prime} \\
1^{\prime} 8^{\prime \prime} \\
1^{\prime} 10^{\prime \prime} \\
2^{\prime} 0^{\prime \prime}\end{array}$ & $\begin{array}{l}1^{\prime} 0^{\prime \prime} \times 2^{\prime} 10^{\prime \prime} \\
1^{\prime} 2^{\prime \prime} \\
1^{\prime} 4^{\prime \prime} \\
1^{\prime} 6^{\prime \prime} \\
1^{\prime} 8^{\prime \prime} \\
1^{\prime} 10^{\prime \prime} \\
2^{\prime} 0^{\prime \prime} \\
1^{\prime} 0^{\prime \prime} \times 3^{\prime} 0^{\prime \prime} \\
1^{\prime} 2^{\prime \prime} \\
1^{\prime} 4^{\prime \prime} \\
1^{\prime} 6^{\prime \prime} \\
1^{\prime} 8^{\prime \prime} \\
1^{\prime} 10^{\prime \prime} \\
2^{\prime} 0^{\prime \prime} \\
1^{\prime} 0^{\prime \prime} \times 3^{\prime} 6^{\prime \prime} \\
1^{\prime} 2^{\prime \prime} \\
1^{\prime} 4^{\prime \prime} \\
1^{\prime} 6^{\prime \prime} \\
1^{\prime} 8^{\prime \prime} \\
1^{\prime} 10^{\prime \prime} \\
2^{\prime} 0^{\prime \prime} \\
1^{\prime} 0^{\prime \prime} \times 4^{\prime} 0^{\prime \prime} \\
1^{\prime} 2^{\prime \prime} \\
1^{\prime} 4^{\prime \prime} \\
1^{\prime} 6^{\prime \prime} \\
1^{\prime} 8^{\prime \prime} \\
1^{\prime} 10^{\prime \prime} \\
2^{\prime} 0^{\prime \prime} \\
1^{\prime} 0^{\prime \prime} \times 4^{\prime \prime} 6^{\prime \prime} \\
1^{\prime} 2^{\prime \prime} \\
1^{\prime} 4^{\prime \prime} \\
1^{\prime} 6^{\prime \prime} \\
1^{\prime} 8^{\prime \prime} \\
1^{\prime} 10^{\prime \prime} \\
2^{\prime} 0^{\prime \prime}\end{array}$ & $\begin{array}{l}1^{\prime} 0^{\prime \prime} \times 5^{\prime} 0^{\prime \prime} \\
1^{\prime} 2^{\prime \prime} \\
1^{\prime} 4^{\prime \prime} \\
1^{\prime} 6^{\prime \prime} \\
1^{\prime} 8^{\prime \prime} \\
1^{\prime} 10^{\prime \prime} \\
2^{\prime} 0^{\prime \prime} \\
1^{\prime} 0^{\prime \prime} \times 5^{\prime} 6^{\prime \prime} \\
1^{\prime} 2^{\prime \prime} \\
1^{\prime} 4^{\prime \prime} \\
1^{\prime} 6^{\prime \prime} \\
1^{\prime} 8^{\prime \prime} \\
1^{\prime} 10^{\prime \prime} \\
2^{\prime} 0^{\prime \prime} \\
1^{\prime} 0^{\prime \prime} \times 5^{\prime} 8^{\prime \prime} \\
1^{\prime} 2^{\prime \prime} \\
1^{\prime} 4^{\prime \prime} \\
1^{\prime} 6^{\prime \prime} \\
1^{\prime} 8^{\prime \prime} \\
1^{\prime} 10^{\prime \prime} \\
2^{\prime} 0^{\prime \prime} \\
1^{\prime} 0^{\prime \prime} \times 5^{\prime} 10^{\prime \prime} \\
1^{\prime} 2^{\prime \prime} \\
1^{\prime} 4^{\prime \prime} \\
1^{\prime} 6^{\prime \prime} \\
1^{\prime} 8^{\prime \prime} \\
1^{\prime} 10^{\prime \prime} \\
2^{\prime} 0^{\prime \prime} \\
1^{\prime} 0^{\prime \prime} \times 6^{\prime} 0^{\prime \prime} \\
1^{\prime} 2^{\prime \prime} \\
1^{\prime} 4^{\prime \prime} \\
1^{\prime} 6^{\prime \prime} \\
1^{\prime} 8^{\prime \prime} \\
1^{\prime} 10^{\prime \prime} \\
2^{\prime} 0^{\prime \prime}\end{array}$ & $\begin{array}{l}2^{\prime} 0^{\prime \prime} \times 6^{\prime} 0^{\prime \prime} \\
2^{\prime} 4^{\prime \prime} \\
2^{\prime} 6^{\prime \prime} \\
2^{\prime} 8^{\prime \prime} \\
3^{\prime} 0^{\prime \prime} \\
1^{\prime} 6^{\prime \prime} \times 6^{\prime} 6^{\prime \prime} \\
1^{\prime} 8^{\prime \prime \prime} \\
2^{\prime} 0^{\prime \prime} \\
2^{\prime} 2^{\prime \prime} \\
2^{\prime} 4^{\prime \prime} \\
2^{\prime} 6^{\prime \prime} \\
2^{\prime} 8^{\prime \prime} \\
3^{\prime} 0^{\prime \prime} \\
1^{\prime} 6^{\prime \prime} \times 6^{\prime} 8^{\prime \prime} \\
1^{\prime} 8^{\prime \prime} \\
2^{\prime} 0^{\prime \prime} \\
2^{\prime} 2^{\prime \prime} \\
2^{\prime} 4^{\prime \prime} \\
2^{\prime} 6^{\prime \prime} \\
2^{\prime} 8^{\prime \prime} \\
2^{\prime} 10^{\prime \prime} \\
3^{\prime} 0^{\prime \prime} \\
3^{\prime} 4^{\prime \prime} \\
3^{\prime} 6^{\prime \prime} \\
1^{\prime \prime} 6^{\prime \prime} \times 6^{\prime} 10^{\prime \prime} \\
1^{\prime} 8^{\prime \prime} \\
2^{\prime} 0^{\prime \prime} \\
2^{\prime} 2^{\prime \prime} \\
2^{\prime} 4^{\prime \prime} \\
2^{\prime} 6^{\prime \prime} \\
2^{\prime} 8^{\prime \prime} \\
2^{\prime} 10^{\prime \prime} \\
3^{\prime} 0^{\prime \prime}\end{array}$ & $\begin{array}{l}1^{\prime} 6^{\prime \prime} \times 7^{\prime} 0^{\prime \prime} \\
1^{\prime} 8^{\prime \prime} \\
2^{\prime} 0^{\prime \prime} \\
2^{\prime} 2^{\prime \prime} \\
2^{\prime} 4^{\prime \prime} \\
2^{\prime} 6^{\prime \prime} \\
2^{\prime} 8^{\prime \prime} \\
2^{\prime} 10^{\prime \prime} \\
3^{\prime} 0^{\prime \prime} \\
3^{\prime} 4^{\prime \prime} \\
3^{\prime} 6^{\prime \prime} \\
2^{\prime} 0^{\prime \prime} \times 7^{\prime} 6^{\prime \prime} \\
2^{\prime} 6^{\prime \prime} \\
2^{\prime} 8^{\prime \prime} \\
2^{\prime} 10^{\prime \prime} \\
3^{\prime} 0^{\prime \prime} \\
3^{\prime} 4^{\prime \prime} \\
3^{\prime} 6^{\prime \prime} \\
2^{\prime \prime} 6^{\prime \prime} \times 8^{\prime} 0^{\prime \prime} \\
2^{\prime} 8^{\prime \prime} \\
2^{\prime} 10^{\prime \prime} \\
3^{\prime} 0^{\prime \prime} \\
3^{\prime} 4^{\prime \prime} \\
3^{\prime} 6^{\prime \prime} \\
\end{array}$ & $\begin{array}{l}1^{\prime} 0^{\prime \prime} \times 6^{\prime} 8^{\prime \prime} \\
1^{\prime} 2^{\prime \prime} \\
1^{\prime} 4^{\prime \prime} \\
1^{\prime} 6^{\prime \prime} \\
1^{\prime} 0^{\prime \prime} \times 6^{\prime} 10^{\prime \prime} \\
1^{\prime} 2^{\prime \prime} \\
1^{\prime} 4^{\prime \prime} \\
1^{\prime} 6^{\prime \prime} \\
1^{\prime} 0^{\prime \prime} \times 7^{\prime} 0^{\prime \prime} \\
1^{\prime} 2^{\prime \prime} \\
1^{\prime} 4^{\prime \prime} \\
1^{\prime} 6^{\prime \prime} \\
1^{\prime} 0^{\prime \prime} \times 7^{\prime} 6^{\prime \prime} \\
1^{\prime} 2^{\prime \prime} \\
1^{\prime}{ }^{\prime \prime} \\
1^{\prime} 6^{\prime \prime} \\
1^{\prime} 0^{\prime \prime} \times 8^{\prime} 0^{\prime \prime} \\
1^{\prime} 2^{\prime \prime} \\
1^{\prime} 4^{\prime \prime} \\
1^{\prime} 6^{\prime \prime}\end{array}$ \\
\hline $\begin{array}{l}\text { Standard } \\
\text { sizes, } \\
\text { garage } \\
\text { doors }\end{array}$ & Standard size & lind or summer & $\begin{array}{l}\text { Standard sizes } \\
\text { and stor }\end{array}$ & $\begin{array}{l}\text { s, combination } \\
\text { rm doors }\end{array}$ & $\begin{array}{l}\text { Standard } \\
\text { sizes, } \\
\text { toilet doors }\end{array}$ \\
\hline $\begin{array}{l}2^{\prime} 0^{\prime \prime} \times 7^{\prime} 0^{\prime \prime} \\
2^{\prime} 4^{\prime \prime} \\
2^{\prime} 6^{\prime \prime} \\
2^{\prime} 8^{\prime \prime} \\
3^{\prime} 0^{\prime \prime} \\
3^{\prime} 6^{\prime \prime} \\
3^{\prime} 9^{\prime \prime} \\
4^{\prime} 0^{\prime \prime} \\
2^{\prime \prime} \\
2^{\prime \prime} 0^{\prime \prime} \times 7^{\prime} 6^{\prime \prime} \\
2^{\prime} 4^{\prime \prime} \\
2^{\prime} 6^{\prime \prime} \\
2^{\prime} 8^{\prime \prime} \\
3^{\prime} 0^{\prime \prime} \\
3^{\prime} 6^{\prime \prime} \\
3^{\prime} 9^{\prime \prime} \\
4^{\prime} 0^{\prime \prime} \\
2^{\prime \prime} 0^{\prime \prime} \times 8^{\prime} 0^{\prime \prime} \\
2^{\prime} 4^{\prime \prime \prime} \\
2^{\prime} 6^{\prime \prime} \\
2^{\prime} 8^{\prime \prime} \\
3^{\prime} 0^{\prime \prime} \\
3^{\prime} 6^{\prime \prime} \\
3^{\prime} 9^{\prime \prime} \\
4^{\prime} 0^{\prime \prime}\end{array}$ & $\begin{array}{l}2^{\prime} 0^{\prime \prime} \times 4^{\prime} 6^{\prime \prime} \\
2^{\prime}{ }^{\prime \prime} \\
2^{\prime} 6^{\prime \prime} \\
2^{\prime} 0^{\prime \prime} \times 5^{\prime} 0^{\prime \prime} \\
2^{\prime} 4^{\prime \prime} \\
2^{\prime} 6^{\prime \prime} \\
2^{\prime} 0^{\prime \prime} \times 5^{\prime} 6^{\prime \prime} \\
2^{\prime} 4^{\prime \prime} \\
2^{\prime} 6^{\prime \prime} \\
2^{\prime} 0^{\prime \prime} \times 6^{\prime} 0^{\prime \prime} \\
2^{\prime} 4^{\prime \prime} \\
2^{\prime} 6^{\prime \prime} \\
2^{\prime} 8^{\prime \prime} \\
2^{\prime} 10^{\prime \prime} \\
3^{\prime} 0^{\prime \prime}\end{array}$ & $\begin{array}{l}2^{\prime} 6^{\prime \prime} \times 6^{\prime} 6^{\prime \prime} \\
2^{\prime} 8^{\prime \prime} \\
2^{\prime} 10^{\prime \prime} \\
3^{\prime} 0^{\prime \prime} \\
2^{\prime} 6^{\prime} \times 6^{\prime} 8^{\prime \prime} \\
2^{\prime} 8^{\prime \prime} \\
2^{\prime} 10^{\prime \prime} \\
3^{\prime} 0^{\prime \prime} \\
2^{\prime} 10^{\prime \prime} \times 6^{\prime} 10^{\prime \prime} \\
2^{\prime} 8^{\prime \prime} \times 7^{\prime} 0^{\prime \prime} \\
2^{\prime} 10^{\prime \prime} \\
3^{\prime} 0^{\prime \prime}\end{array}$ & $\begin{array}{l}2^{\prime} 6^{\prime \prime} \mathrm{x} \\
2^{\prime} 6^{\prime \prime} \mathrm{x} \\
2^{\prime} 8^{\prime \prime} \\
2^{\prime} 10^{\prime \prime} \\
3^{\prime} 0^{\prime \prime} \\
2^{\prime} 10^{\prime \prime} \mathrm{x} \\
2^{\prime} 6^{\prime \prime} \mathrm{x} \\
2^{\prime} 8^{\prime \prime} \\
2^{\prime} 10^{\prime \prime} \\
3^{\prime} 0^{\prime \prime}\end{array}$ & $\begin{array}{l}6^{\prime} 7^{\prime \prime} \\
6^{\prime} 9^{\prime \prime} \\
\times 6^{\prime} 11^{\prime \prime} \\
7^{\prime} 1^{\prime \prime}\end{array}$ & $\begin{array}{l}2^{\prime} 0^{\prime \prime} \times 4^{\prime} 0^{\prime \prime} \\
2^{\prime} 4^{\prime \prime} \\
2^{\prime} 6^{\prime \prime} \\
2^{\prime} 0^{\prime \prime} \times 4^{\prime} 6^{\prime \prime} \\
2^{\prime} 4^{\prime \prime} \\
2^{\prime} 6^{\prime \prime} \\
2^{\prime} 0^{\prime \prime} \times 5^{\prime} 0^{\prime \prime} \\
2^{\prime} 4^{\prime \prime} \\
2^{\prime} 6^{\prime \prime} \\
2^{\prime} 0^{\prime \prime} \times 5^{\prime} 6^{\prime \prime} \\
2^{\prime} 4^{\prime \prime} \\
2^{\prime} 6^{\prime \prime} \\
2^{\prime} 0^{\prime \prime} \times 6^{\prime} 0^{\prime \prime} \\
2^{\prime} 4^{\prime \prime} \\
2^{\prime} 6^{\prime \prime}\end{array}$ \\
\hline
\end{tabular}




\section{INTERIOR DOORS}

N.D. 100

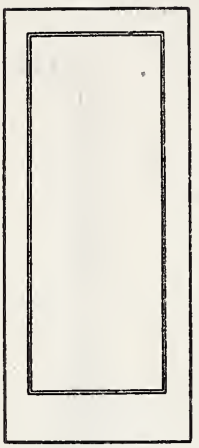

ONE PANEL

Stiles and top rail

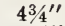

Bottom rail

3-ply plywood flat panel, Sticking: Standard.

N. D. 102

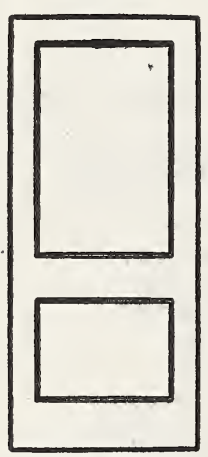

TWO PANEL

Stiles and top rail

Lock rail

3-ply plywood flat panels. Sticking: Standard.
N.D. 101

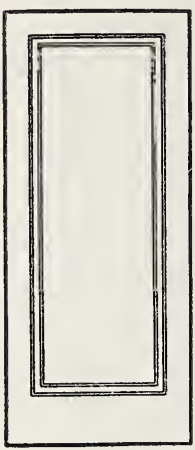

INNER FRAME

Stiles and top rail................... $414^{\prime \prime}$ face Bottom rail $91 / 4^{\prime \prime}$ or $91 / 2^{\prime \prime}$ face

3-ply plywood flat panel. Sticking: P\&G or Standard.

N.D. 103

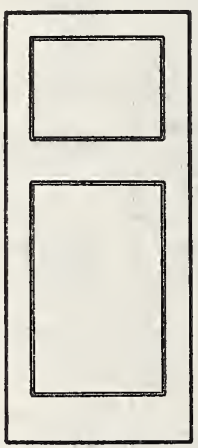

TWO REVERSE PANEL

Stiles and top rail _............. $434^{\prime \prime}$

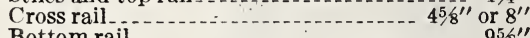

Bottom rail . . .

3-ply plywood flat panels. Sticking: Standard. 
INTERIOR DOORS-Continued

N.D. 104

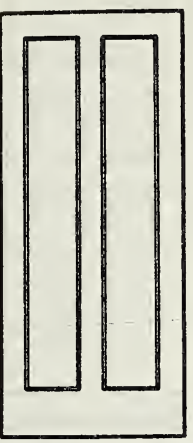

TWO VERTICAL PANEL

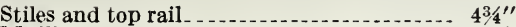

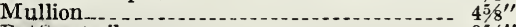
Bottom rail

3-ply plywood flat panels. Sticking: Standard.

N.D. 108

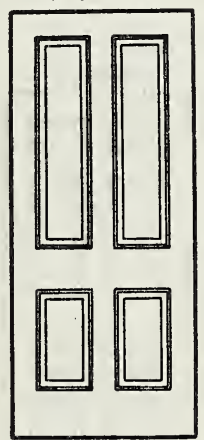

FOUR PANEL

Stiles and top rail. . Lock rail.

Muntins .

Bottom rail

Raised panels 2 sides. Can also be furnished with 3-ply plywood flat panels, if desired. Sticking: Standard.
N.D. 105

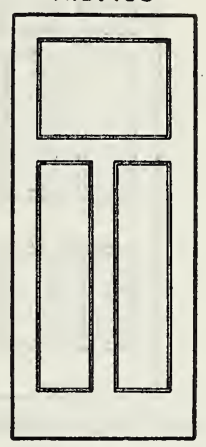

THREE PANEL

Stiles and top rail Cross rail and mullion 3-ply plywood flat panels. Sticking: Standard.

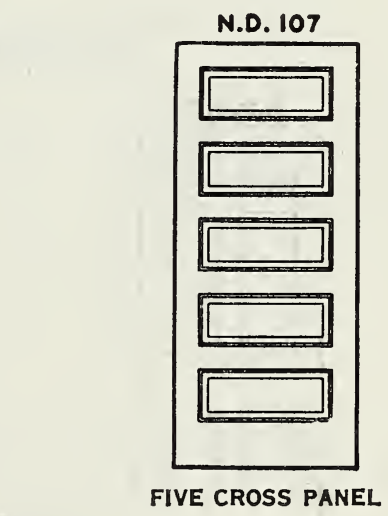

Stiles and top rail

(2) $958^{\prime \prime}$

Raised panels 2 sides. Can also be furnished with 3-ply plywood flat panels, if desired. Sticking: 'Standard.

Doors $7^{\prime} 6^{\prime \prime}$ and $8^{\prime}$ high are made with 6 cross panels. 
INTERIOR DOORS-Continued

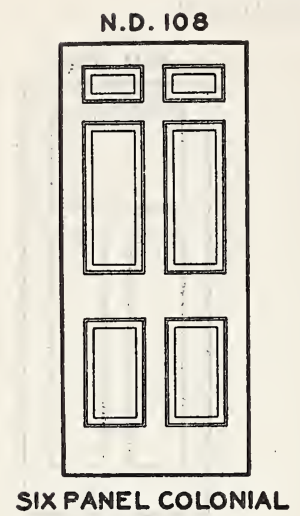

Stiles and top rail Lock rail _. Intermediate rail and mullions..... $37 / 8^{\prime \prime}$ or $458^{\prime \prime}$ Bottom rail ............................ $8^{\prime \prime}$ or $958^{\prime \prime}$ Height of top panels over-all........... $71 / 8^{\prime \prime}$

Raised panels 2 sides. Can also be furnished with 3-ply plywood flat panels. Sticking: Standard.

Doors $1^{\prime} 8^{\prime \prime}$ and narrower are made 1 panel wide.
N.D. 109

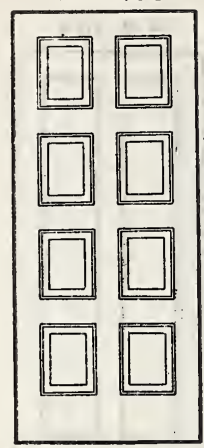

EIGHT PANEL

Stiles and top rail $\ldots \ldots \ldots$. Intermediate rails and mullions Bottom rail ........................... $95 \delta^{\prime \prime}$

Raised panels 2 sides. Can also be furnished with 3-ply plywood flat panels. Sticking: Standard.

Doors $1^{\prime} 8^{\prime \prime}$ and narrower are made 1 panel wide.

\section{EXTERIOR DOORS}

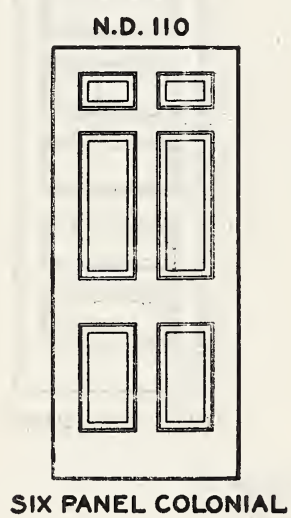

Stiles and top rail $\ldots$ Lock rail........................... 8 or $958^{\prime \prime}$ Intermediate rail and mullions . . ......... $538^{\prime \prime}$ Bottom rail.............. $8^{\prime \prime}$ or $958^{\prime \prime}$ Panel thickness................... $3 / 4^{\prime \prime}$ Height of top panels over-all............... $71 / 8^{\prime \prime}$

Raised panels 2 sides. Sticking: Standard.
N.D. 112

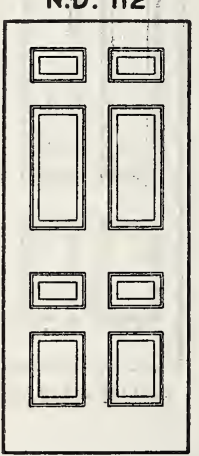

EIGHT PANEL COLONIAL

Stiles and top rail _. _..._............... $51 / 2^{\prime \prime}$ Lock rail ............................. $8^{\prime \prime}$ or $958^{\prime \prime}$ Intermediate rails and mullions.......... $538^{\prime \prime}$ Bottom rail.............. $8^{\prime \prime}$ or $958^{\prime \prime}$

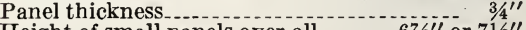

Height of small panels over-all...... $67 \% 8^{\prime \prime}$ or $71 / 8^{\prime \prime}$

Raised panels 2 sides. Sticking: Standard. 
EXTERIOR DOORS-Continued
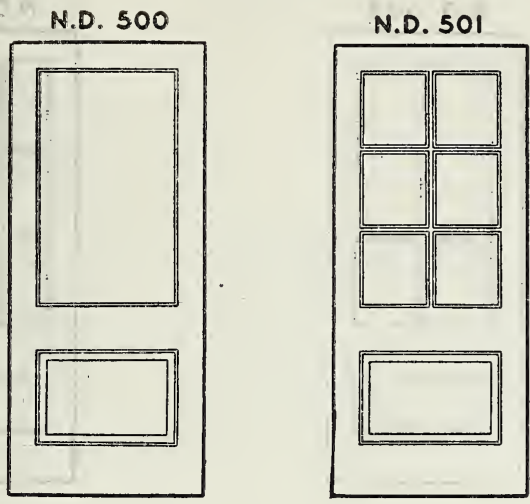

N.D. 502

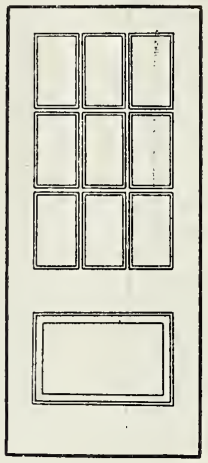

N.D. 505

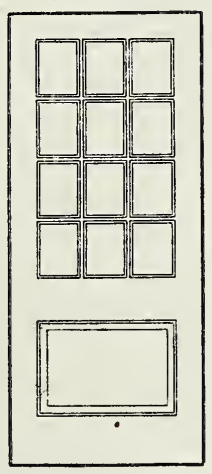

N.D. 506

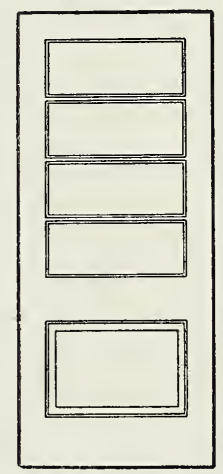

Stiles and top rail

Lock rail
Bottom rail.........

Raised panel 2 sides. Can also be furnished with 3-ply plywood flat panel, if desired. Sticking: Standard.

Door No.

N. D. 500

N. D. 501

N. D. 502

N. D. 505
Approximate glass size

Size of door
$3^{\prime} 0^{\prime \prime} \times 6^{\prime} 8^{\prime \prime}$

Size of door
$3^{\prime} 0^{\prime \prime} \times 7^{\prime} 0^{\prime \prime}$ Size of door
$2^{\prime} 8^{\prime \prime} \times 6^{\prime} 8^{\prime \prime}$

$22^{\prime \prime} \times 40^{\prime \prime}$

$10^{3} 4^{\prime \prime} \times 13^{\prime \prime}$

$7^{\prime \prime} \times 13^{\prime \prime}$

$7^{\prime \prime} \times 9^{5} 8^{\prime \prime}$

$22^{\prime \prime} \times 958^{\prime \prime}$

$26^{\prime \prime} \times 40^{\prime \prime}$

$12^{3} 4^{\prime \prime} \times 13^{\prime \prime}$

$8516^{\prime \prime} \times 13^{\prime \prime}$

$8516^{\prime \prime} \times 958^{\prime \prime}$

$26^{\prime \prime} \times 95 / 8^{\prime \prime}$ $26^{\prime \prime} \times 44^{\prime \prime}$

$1234^{\prime \prime} \times 145 / 16^{\prime \prime}$

$8516^{\prime \prime} \times 145 / 16^{\prime \prime}$

$8316^{\prime \prime} \times 105 / 3^{\prime \prime}$

$26^{\prime \prime} \times 10 \% / 8 \prime$

Beads for glass included. 


\section{EXTERIOR DOORS-Continued}

N.D. 507

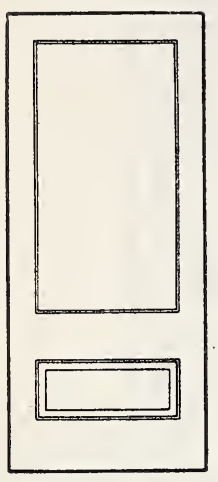

N.D. 510

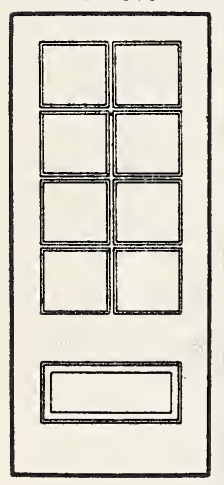

N.D. 508

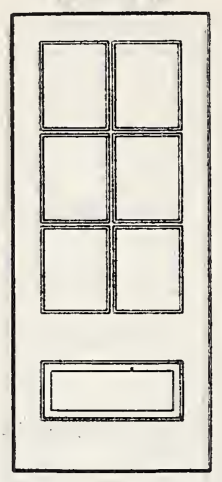

N.D. 511

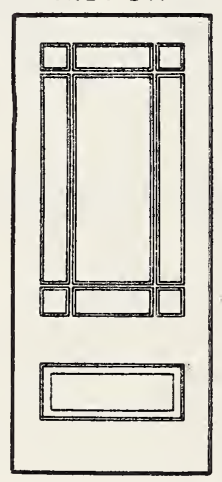

N.D. 509

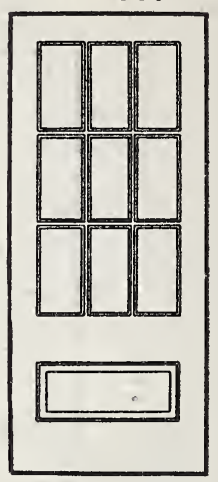

N.D. 512

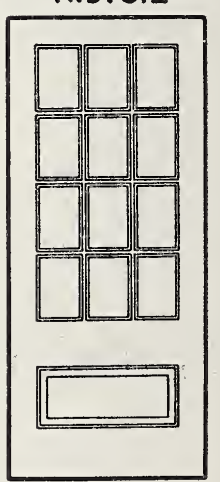

Stiles and top rail

(1) $512^{\prime \prime}$

Lock rail top rail

Bottom rail.

Raised panel 2 sides. Can also be furnished with 3-ply plywood flat panel, if desired. Sticking: Standard.

Door No.

N. D. 507

N. D. 508

N. D. 510

N. D. 511

N. D. 512

\section{Approximate glass size}

Size of door $2^{\prime} 8^{\prime \prime} \times 6^{\prime} 8^{\prime \prime}$

$22^{\prime \prime} \times 46^{\prime \prime}$

$1034^{\prime \prime} \times 15^{\prime \prime}$

$7 " \prime \times 15^{\prime \prime}$

$1034^{\prime \prime} \times 1138^{\prime \prime}$

$5^{\prime \prime} \times 5^{\prime \prime *}$

$7^{\prime \prime} \times 114^{\prime \prime}$
Size of door

$26^{\prime \prime} \times 46^{\prime \prime}$

$1234^{\prime \prime} \times 15^{\prime \prime}$

$85 / 16^{\prime \prime} \times 15^{\prime \prime}$

$123 / 4^{\prime \prime} \times 111 / 8^{\prime \prime}$

$5^{\prime \prime} \times 5^{\prime \prime *}$ $3^{\prime} 0^{\prime \prime} \times 6^{\prime} 8^{\prime \prime}$
Size of door $3^{\prime} 0^{\prime \prime} \times 7^{\prime} 0^{\prime \prime}$

$26^{\prime \prime} \times 50^{\prime \prime}$ $1234^{\prime \prime} \times 1651^{\prime \prime}$ $85 / 16^{\prime \prime} \times 16516^{\prime \prime}$ $12{ }^{3 / 4} \times 1218^{\prime \prime}$ $5^{\prime \prime} \times 5^{\prime \prime *}$ $8{ }^{\prime \prime} 6^{\prime \prime} \times 121 / 8^{\prime \prime}$

Beads for glass included.

* Corner lights. 


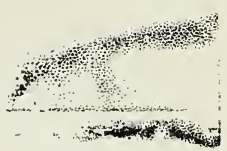

EXTERIOR DOORS-Continued

N.D. 513

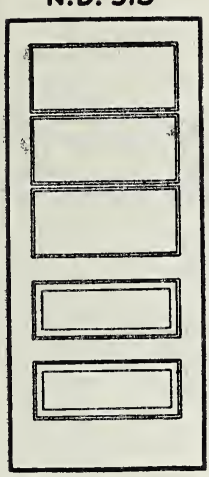

N.D. 517

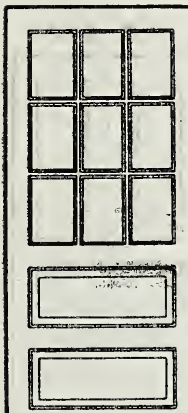

N.D. 514

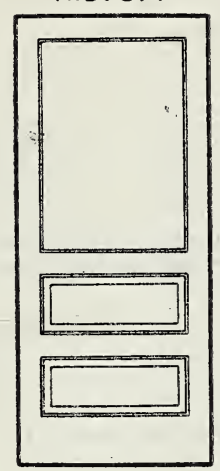

N.D. 515

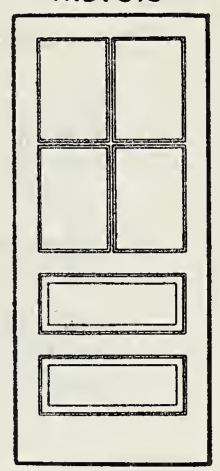

N.D. 516

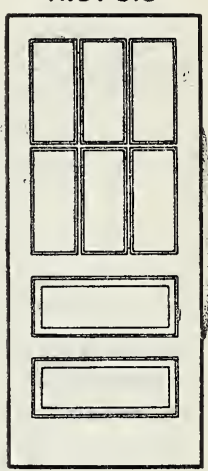

Stiles and top rail

Lock rail

Intermediate rail

Bottom rail

Raised panels 2 sides. Can also be furnished with 3 -ply plywood flat panels, if desired. Sticking: Standard.

Door No.

N. D. 513

N. D. 515

N. D. 516

N. D. 517

N. D. 519

N. D. 521
N.D. 521

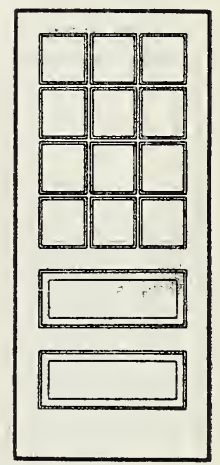

$434^{\prime \prime}$

N.D. 519

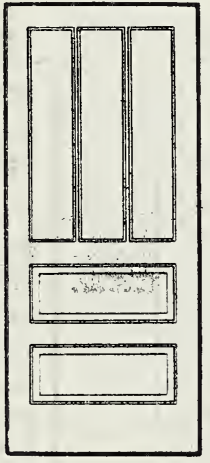

Approximate glass size

Size of door Size of door $3^{\prime} 0^{\prime \prime} \times 6^{\prime} 8^{\prime \prime} \quad 3^{\prime} 0^{\prime \prime} \times 7^{\prime} 0^{\prime \prime}$

$2712^{\prime \prime} \times 1156^{\prime \prime} \quad 2712^{\prime \prime} \times 13^{\prime \prime}$ $271^{\prime \prime} \times 36^{\prime \prime} \quad 2712^{\prime \prime} \times 40^{\prime \prime}$ $131^{1}$ " $^{\prime \prime} \times 1734^{\prime \prime} \quad 131 / 2^{\prime \prime} \times 193$, $^{\prime \prime}$

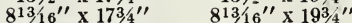
$8136^{\prime \prime} \times 115,8$ " $813 / 16^{\prime \prime} \times 36^{\prime \prime}$ $813 / 16^{\prime \prime} \times 88^{5} / 8^{\prime \prime}$

$81316^{\prime \prime} \times 193 / 4$ $813 / 16^{\prime \prime} \times 13^{\prime \prime}$ $81316^{\prime \prime} \times 40^{\prime \prime}$ $813 / 16^{\prime \prime} \times 95 / 8^{\prime \prime}$

Beads for glass included. 


\section{EXTERIOR DOORS-Continued}
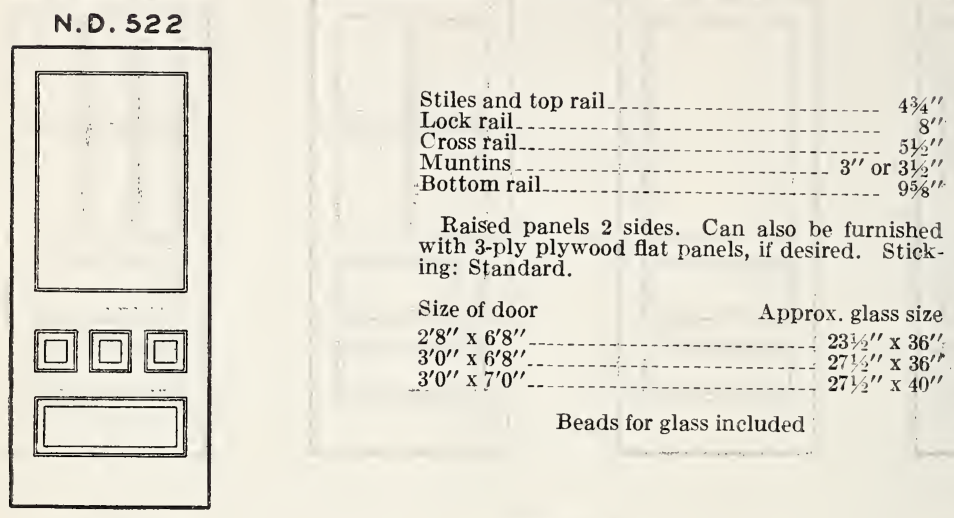

Raised panels 2 sides. Can also be furnished with 3-ply plywood flat panels, if desired. Sticking: Standard.

\begin{abstract}
Size of door Approx. glass size $2^{\prime} 8^{\prime \prime} \times 6^{\prime} 8^{\prime \prime} \ldots \ldots \ldots 231 / 2{ }^{\prime \prime} \times 36^{\prime \prime}$ $3^{\prime} 0^{\prime \prime} \times 6^{\prime} 8^{\prime \prime} \ldots \ldots \ldots \ldots+27{ }_{2}^{\prime \prime} \times 36^{\prime \prime}$ $3^{\prime} 0^{\prime \prime}$ x $7^{\prime} 0^{\prime \prime} \ldots \ldots$
\end{abstract}

Beads for glass included
N.D. 530

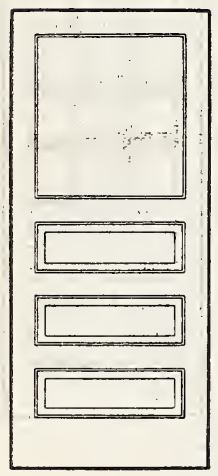

N.D. 531

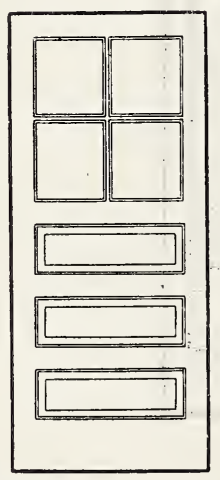

N.D. 532

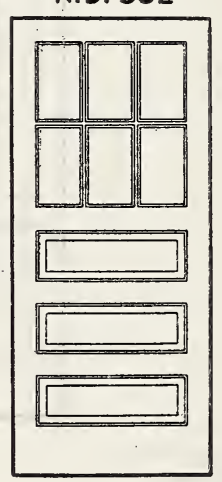

N.D. 533

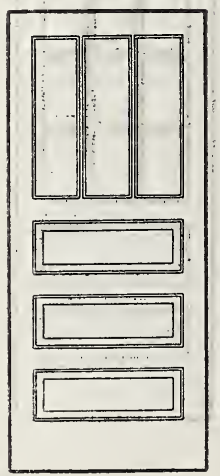

Stiles and top rail.

Stiles and top rail
Cross rails
Bottom rail

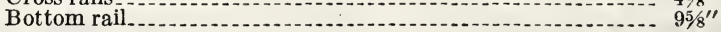

Raised panels 2 sides. Can also be furnished with 3-ply plywood flat panels, if desired. Sticking: Standard.

Door No.

N. D. 530

N. D. 531

N. D. 532

N. D. 533

\section{Size of door} $2^{\prime} 6^{\prime \prime} \times 6^{\prime} 6^{\prime \prime}$

$211 / 2 " \times 24^{\prime \prime}$

$101 / 2^{\prime \prime} \times 11^{3 / 4^{\prime \prime}}$ $613 / 6^{\prime \prime} \times 11^{3} 4^{\prime \prime}$ $61316^{\prime \prime} \times 24^{\prime \prime}$
Approximate glass size

\begin{tabular}{|c|c|}
\hline $\begin{array}{l}\text { Size of door } \\
2^{\prime} 8^{\prime \prime} \times 6^{\prime} 8^{\prime \prime}\end{array}$ & $\begin{array}{l}\text { Size of door } \\
3^{\prime} 0^{\prime \prime} \times 6^{\prime} 8^{\prime \prime}\end{array}$ \\
\hline 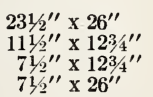 & 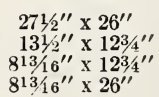 \\
\hline
\end{tabular}

Size of door $3^{\prime} 0^{\prime \prime} \times 7^{\prime} 0^{\prime \prime}$ $2712^{\prime \prime} \times 30^{\prime \prime}$ $131,2^{\prime \prime} \times 1434^{\prime \prime}$ $813 / 16^{\prime \prime} \times 1434^{\prime \prime}$ $813 / 16^{\prime \prime} \times 30^{\prime \prime}$

Beads for glass included. 


\section{EXTERIOR DOORS-Continued}

N.D. 536

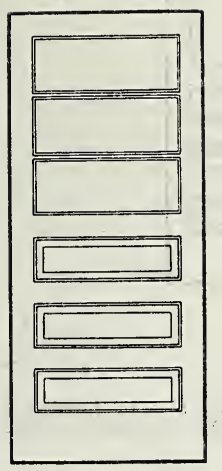

N.D. 539

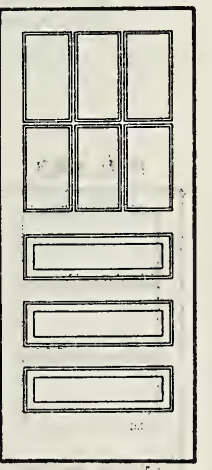

N.D. 537

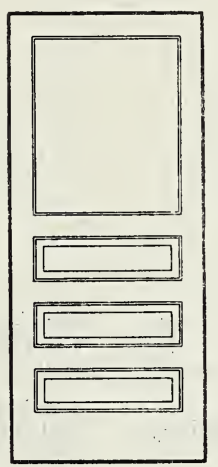

N.D. 540

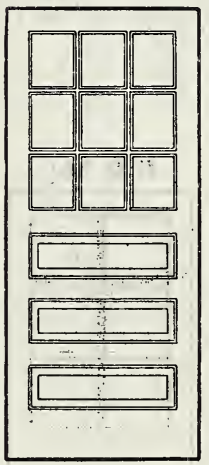

N.D. 538

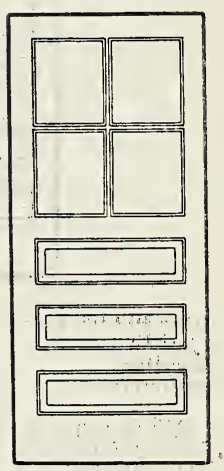

N.D. 542
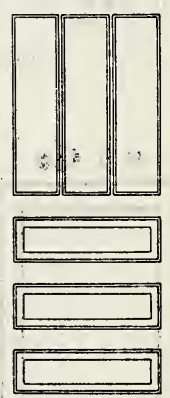

Stiles and top rail

Cross rails

Bottom rail

Raised panels 2 sides. Can also be furnished with 3-ply plywood flat panels, if desired. Sticking: Standard.

\section{Door No.}

N. D. 536

N. D. 537

N. D. 538

N. D. 539

N. D. 540

N. D. 542
Approximate glass size

\begin{tabular}{|c|c|}
\hline $\begin{array}{l}\text { Size of door } \\
2^{\prime} 8^{\prime \prime} \times 6^{\prime} 8^{\prime \prime}\end{array}$ & $\begin{array}{l}\text { Size of door } \\
3^{\prime} 0^{\prime \prime} \times 6^{\prime} 8^{\prime \prime}\end{array}$ \\
\hline $\begin{array}{l}231 / 2^{\prime \prime} \times 958^{\prime \prime} \\
231 / 2^{\prime \prime} \times 30^{\prime \prime} \\
111 / 2^{\prime \prime} \times 143 / 4^{\prime \prime} \\
71 / 2^{\prime \prime} \times 1434^{\prime \prime} \\
71 / 22^{\prime \prime} \times 95 \% 8^{\prime \prime} \\
71 / 2^{\prime \prime} \times 30^{\prime \prime}\end{array}$ & 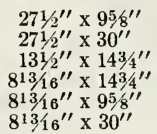 \\
\hline
\end{tabular}

Size of door $3^{\prime} 0^{\prime \prime} \times 7^{\prime} 0^{\prime \prime}$

271 (2) $^{\prime \prime} \times 11^{\prime \prime}$ $2712^{\prime \prime} \times 34^{\prime \prime}$ $131 / 2^{\prime \prime} \times 16^{3} 4^{\prime \prime}$ $813 / 16^{\prime \prime} \times 16^{3} 4^{\prime \prime}$ $813 / 16^{\prime \prime} \times 11^{\prime \prime}$ $8^{13} 16^{\prime \prime} \times 34^{\prime \prime}$

Beads for glass included. 


\section{EXTERIOR DOORS-Continued}

N.D. 544

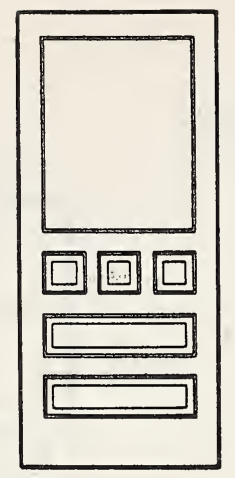

Stiles and top rail.

- $43 / 4$,

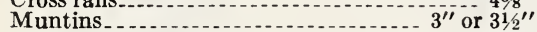

Bottom rail ...............

Raised panels 2 sides. Can also be furnished with 3-ply plywood flat panels, if desired. Sticking: Standard.

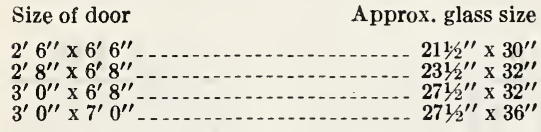

Beads for glass included
N.D. 549

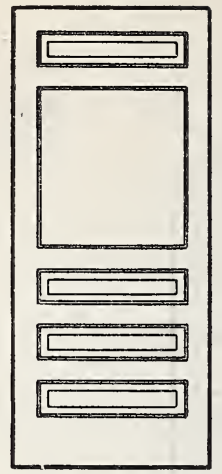

Stiles and top rail

Cross rails

Bottom rail.

$434^{\prime \prime}$ $458^{\prime \prime}$

Raised panels 2 sides. Can also be furnished with 3-ply plywood flat panels, if desired. Sticking: Standard.

Size of door

Approx. glass size $2^{\prime} 6^{\prime \prime} \times 6^{\prime} 6^{\prime \prime} \ldots 211^{\prime \prime} \times 24^{\prime \prime}$

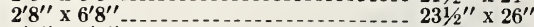

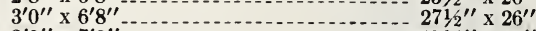

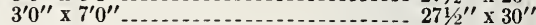

Beads for glass included
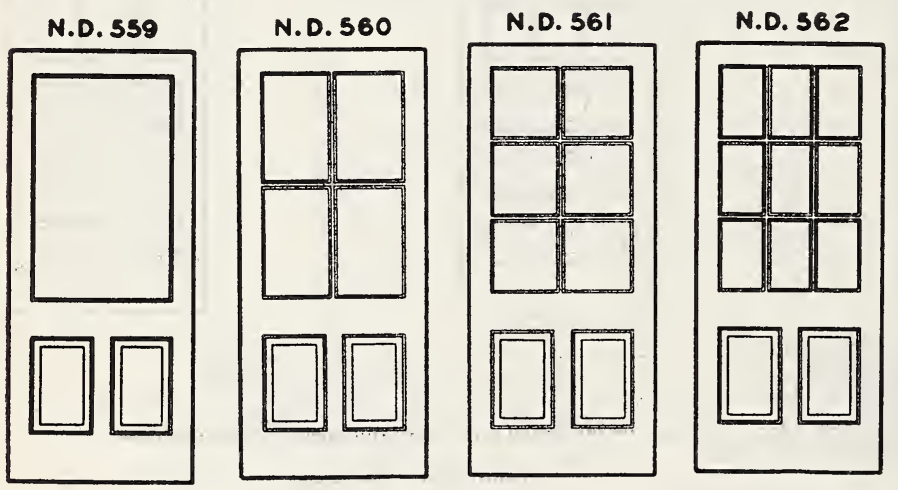

N.D. 563

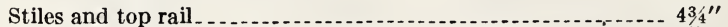

Lock rail . ...

Muntins
Bottom rail

Raised panels 2 sides. Can also be furnished with 3-ply plywood flat panels, if desired. Sticking: Standard.

Door No.

N. D. 559

N. D. 560

N. D. 561

N. D. 562

N. D. 563.

Size of door
$2^{\prime} 6^{\prime \prime} \times 6^{\prime} 6^{\prime \prime}$
$211 / 6^{\prime \prime} \times 38^{\prime \prime}$
$101 / 2^{\prime \prime} \times 184^{\prime \prime}$
$101 / 2 \prime$ × $12516^{\prime \prime}$
$613,16^{\prime \prime} \times 12516^{\prime \prime}$
$211 / 2^{\prime \prime} \times 12516^{\prime \prime}$

Approximate glass size

$\begin{array}{ll}\text { Size of door } & \text { Size of door } \\ 2^{\prime} 8^{\prime \prime} \times 6^{\prime} 8^{\prime \prime} & 3^{\prime} 0^{\prime \prime} \times 6^{\prime} 8^{\prime \prime} \\ 2312^{\prime \prime} \times 40^{\prime \prime} & 2712^{\prime \prime} \times 40^{\prime \prime} \\ 111,2^{\prime \prime} \times 1934^{\prime \prime} & 131,2^{\prime \prime} \times 1934^{\prime \prime} \\ 11 \text {, }^{\prime \prime} \times 13^{\prime \prime} & 131,2^{\prime \prime} \times 13^{\prime \prime} \\ 712^{\prime \prime} \times 13^{\prime \prime} & 813,16^{\prime \prime} \times 13^{\prime \prime} \\ 2312^{\prime \prime} \times 13^{\prime \prime} & 2712^{\prime \prime} \times 13^{\prime \prime}\end{array}$

Size of door $3^{\prime} 0^{\prime \prime} \times 7^{\prime} 0^{\prime \prime}$ $271 / 2 " 1 \times 44^{\prime \prime}$ $1316^{\prime \prime} \times 2134^{\prime \prime}$ $131 / 2^{\prime \prime} \times 145 / 16^{\prime \prime}$ $813 / 6^{\prime \prime} \times 145 / 16^{\prime \prime}$ $2712^{\prime \prime} \times 14 \% 16^{\prime \prime}$

Beads for glass included. 


\section{EXTERIOR DOORS-Continued}

N.D. 567

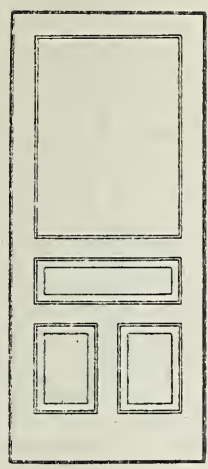

N.D. 568

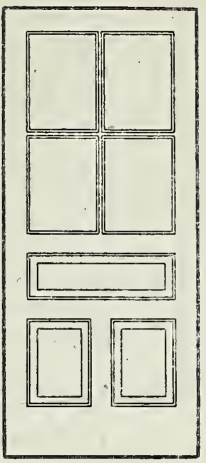

N.D. 569

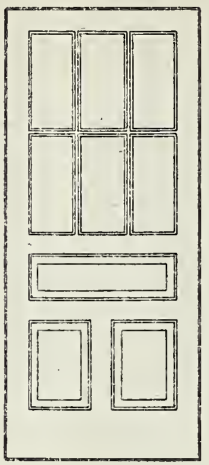

N.D. 570

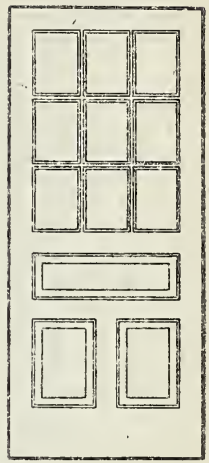

Stiles and top rail $434^{\prime \prime}$

Cross rails. .........

Muntins

Bottom rail

Raised panels 2 sides. Can also be furnished with 3-ply plywood flat panels, if desired. Sticking: Standard.

Door No.

Size of door $2^{\prime} 6^{\prime \prime} \times 6^{\prime} 6^{\prime \prime}$

N. D. 567

N. D. 568

$211 / 2^{\prime \prime} \times 32^{\prime \prime}$

$101 / 2^{\prime \prime} \times 15 \% 4^{\prime \prime}$

$613,16^{\prime \prime} \times 1534^{\prime \prime}$

$613 / 16^{\prime \prime} \times 105 / 16^{\prime \prime}$
Approximate glass size

$\begin{array}{rr}\text { Size of door } & \text { Size of door } \\ 2^{\prime} 8^{\prime \prime} \times 6^{\prime} 8^{\prime \prime} & 3^{\prime} 0^{\prime \prime} \times 6^{\prime} 8^{\prime \prime}\end{array}$

$231 / 2^{\prime \prime} \times 34^{\prime \prime}$

$1112^{\prime \prime} \times 1634^{\prime \prime}$

$71 / 2 \prime \prime \times 163 / 4 " \prime$

$71 / 2^{\prime \prime} \times 11^{\prime \prime}$

2716 '

131 2 $^{\prime \prime} \times 16^{3} 4^{\prime \prime}$

$813 / 16^{\prime \prime} \times 163 / 4^{\prime \prime}$

$813 / 16^{\prime \prime} \times 11^{\prime \prime}$

Size of door
$3^{\prime} 0^{\prime \prime} \times 7^{\prime} 0^{\prime \prime}$
$2712^{\prime \prime} \times 38^{\prime \prime}$
$132^{\prime \prime} \times 18^{3} 4^{\prime \prime}$
$81316^{\prime \prime} \times 1834^{\prime \prime}$
$81316^{\prime \prime} \times 12 \% 16^{\prime \prime}$

Size of door $2712^{\prime \prime} \times 38^{\prime \prime}$ $813 / 16^{\prime \prime} \times 1834^{\prime \prime}$

Beads for glass included.
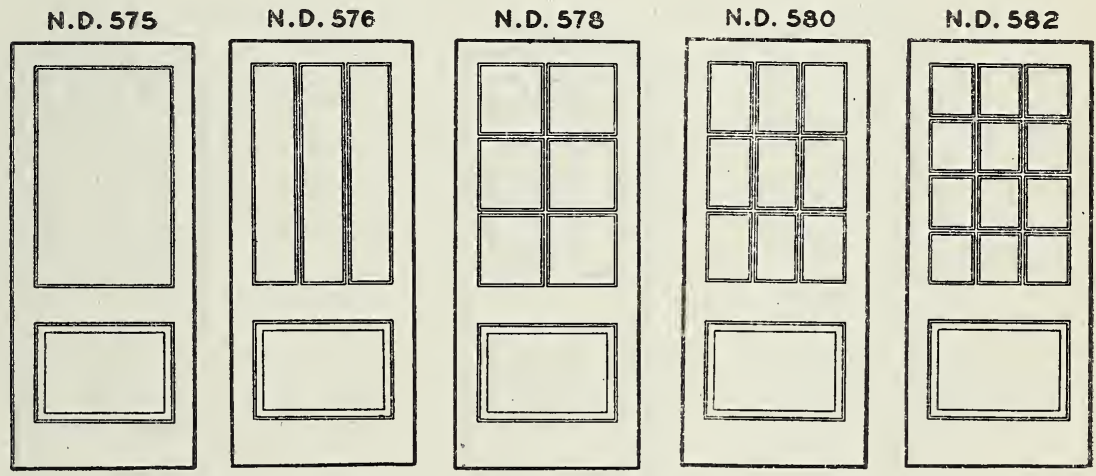

Stiles and top rail Lock rail

Raised panel 2 sides. Can also be furnished with 3-ply plywood flat panels, if desired. Sticking: Standard.

Door No.

Size of door
$2^{\prime} 8^{\prime \prime} \times 6^{\prime} 8^{\prime \prime}$

$231 / 2^{\prime \prime} \times 40^{\prime \prime}$

$71^{\prime \prime} \times 40^{\prime \prime}$

$111^{\prime \prime} \times 13^{\prime \prime}$

N. D. 576

N. D. 578

N. D. 582
$71 / 2^{\prime \prime} \times 13^{\prime \prime}$

$71 / 2 "$ " $\times 95 / 8^{\prime \prime}$

Beads for glass included.
Approximate glass size

Size of door
$3^{\prime} 0^{\prime \prime} \times 6^{\prime} 8^{\prime \prime}$

Size of door

$2712^{\prime \prime} \times 40^{\prime \prime}$

$8^{13} 16^{\prime \prime} \times 40^{\prime \prime}$

$131 / 2^{\prime \prime} \times 13^{\prime \prime}$

$813 / 16^{\prime \prime} \times 13^{\prime \prime}$

$813 / 16^{\prime \prime} \times 95 / 8^{\prime \prime}$ $3^{\prime} 0^{\prime \prime} \times 7^{\prime} 0^{\prime \prime}$

$2712^{\prime \prime} \times 44^{\prime \prime}$ $8131^{\prime \prime} \times 44^{\prime \prime}$

$1312^{\prime \prime} \times 145 / 16^{\prime \prime}$ $813 / 16^{\prime \prime} \times 14516^{\prime \prime}$ $813 / 16^{\prime \prime} \times 10^{\prime} 58^{\prime \prime}$ 


\section{EXTERIOR DOORS-Continued}
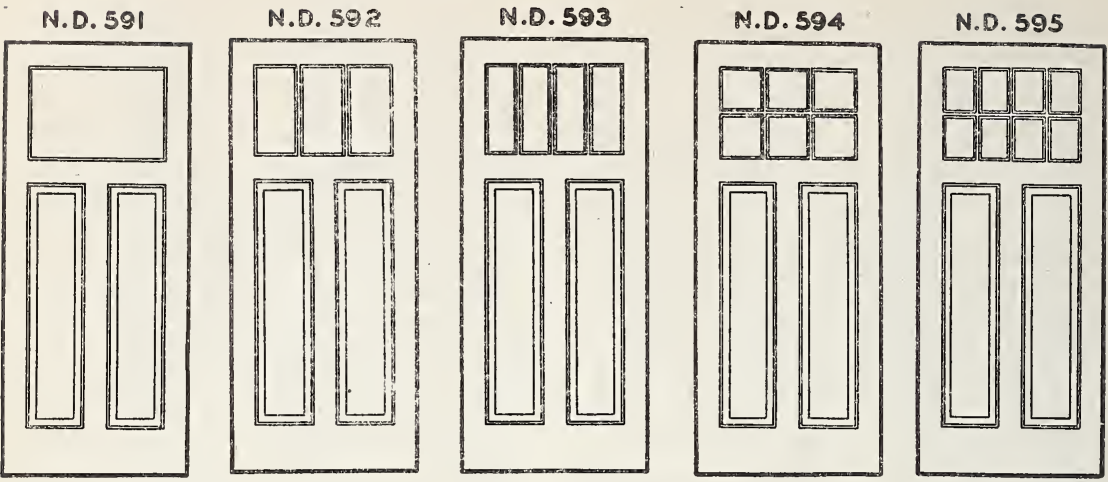

Stiles and top rail

Mullion and cross rail

$43 / 4^{\prime \prime}$ or $51 / 2^{\prime \prime}$

$458^{\prime \prime}$ or $538^{\prime \prime}$

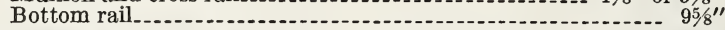

Raised panels 2 sides. Can also be furnished with 3-ply plywood flat panels, if desired. Sticking: Standard.

Door No.

N. D. 591

N. D. 592

N. D. 593

N. D. 594
Approximate glass size (for doors with $51 / 2$ " stiles) Size of door Size of door Size of door $2^{\prime} 8^{\prime \prime} \times 6^{\prime} 8^{\prime \prime} \quad 3^{\prime} 0^{\prime \prime} \times 6^{\prime} 8^{\prime \prime}$ $3^{\prime} 0^{\prime \prime} \times 7^{\prime} 0^{\prime \prime}$ $22^{\prime \prime} \times 18^{\prime \prime}$ $518^{\prime \prime} \times 18^{\prime \prime}$ 7 " $\times 834^{\prime \prime}$ $51, \$^{\prime \prime} \times 83 / 4^{\prime \prime}$

Beads for glass included.

$\begin{array}{rr}26^{\prime \prime} \times 18^{\prime \prime} & 26^{\prime \prime} \times 18^{\prime \prime} \\ 8516^{\prime \prime} \times 18^{\prime \prime} & 85 / 16^{\prime \prime} \times 18^{\prime \prime} \\ 61 / 8^{\prime \prime} \times 18^{\prime \prime} & 61 / 8^{\prime \prime} \times 18^{\prime \prime} \\ 8516^{\prime \prime} \times 834^{\prime \prime} & 85 / 16^{\prime \prime} \times 84^{\prime \prime} \\ 61 / 8^{\prime \prime} \times 84^{\prime \prime} & 61 / 8^{\prime \prime} \times 84^{\prime \prime}\end{array}$

$8516^{\prime \prime} \times 83 / 4 "$
$61 / 8^{\prime \prime} \times 83 / 4^{\prime \prime}$
N.D. 599

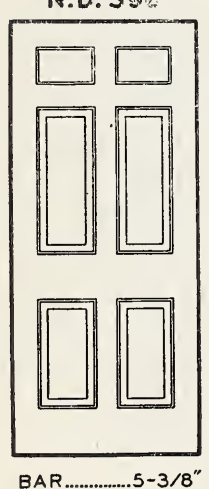

N.D. 597

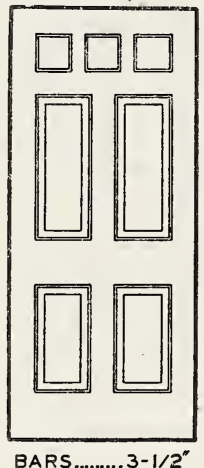

N.D. 598

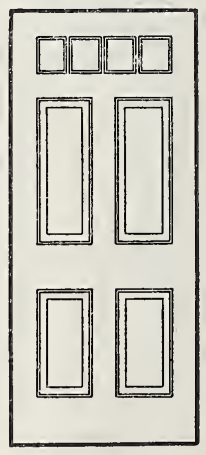

BARS $. .1-1 / 2^{\prime \prime}$

Stiles and top rail Lock rail Top cross rail and mullions. ${ }^{2} \ldots$ Raised panels 2 sides. Sticking: Standard.

Approximate glass size

rNo.

D. 596 .

D. 597

D. 598

Size of door $2^{\prime} 8^{\prime \prime} \times 6^{\prime} 8^{\prime \prime}$

$83 / 4^{\prime \prime} \times 71 / 8^{\prime \prime}$ $534^{\prime \prime} \times 718^{\prime \prime}$

Beads for glass included.

\begin{tabular}{|c|c|}
\hline $\begin{array}{l}\text { Size of door } \\
3^{\prime} 0^{\prime \prime} \times 6^{\prime} 8^{\prime \prime}\end{array}$ & $\begin{array}{l}\text { Size of door } \\
3^{\prime} 0^{\prime \prime} \times 7^{\prime} 0^{\prime \prime}\end{array}$ \\
\hline $\begin{array}{r}103 / 4^{\prime \prime} \times 71 / 8^{\prime \prime} \\
7{ }^{\prime \prime} \times 71 / 8^{\prime \prime} \\
61 / 8^{\prime \prime} \times 71 / 8^{\prime \prime}\end{array}$ & $\begin{array}{r}103 / 4^{\prime \prime} \times 71 / 8^{\prime \prime} \\
7{ }^{\prime \prime} \times 71 / 8^{\prime \prime} \\
61 / 8^{\prime \prime} \times 71 / 8^{\prime \prime}\end{array}$ \\
\hline
\end{tabular}

103/4" x 71/8" $61 / 8^{\prime \prime} \times 71 / 8$ "

$61 / 8^{\prime \prime} \times 71 / 8^{\prime \prime}$ 


\section{EXTERIOR DOORS-Continued}

N.0. 600

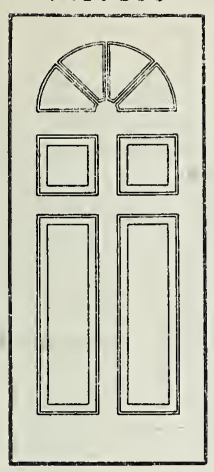

Stiles and top rail Top cross rail

(1) $53 / 8^{\prime \prime}$

\& mullion................. $45 / 8^{\prime \prime}$

Raised panels 2 sides. Can also be furnished with 3-ply plywood flat panels, if desired. Sticking: Standard.

Size of door

Approx. glass opg. $2^{\prime} 8^{\prime \prime} \times 6^{\prime} 8^{\prime \prime} \ldots \ldots \ldots \ldots+\ldots 2^{\prime \prime} \times 11^{\prime \prime}$ $3^{\prime} 0^{\prime \prime} \times 6^{\prime} 8^{\prime \prime}-\ldots$ $3^{\prime} 0^{\prime \prime} \times 7^{\prime} 0^{\prime \prime} \ldots \ldots \ldots$

Beads for glass included.

\section{N.D. 604}

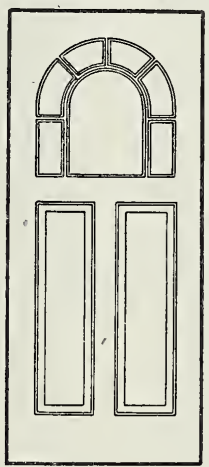

Stiles and top rail Cross rail

$51 / 2^{\prime \prime}$

Mullion -

Bottom rail

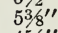
$458^{\prime \prime}$

Raised panels 2 sides. Can also be furnished with 3-ply plywood flat panels, if desired. Sticking: Standard.

\section{Size of door}

Approx. glass opg.

$2^{\prime} 8^{\prime \prime} \times 6^{\prime} 8^{\prime \prime}$

$3^{\prime} 0^{\prime \prime} \times 6^{\prime} 8^{\prime \prime}$ $22^{\prime \prime} \times 22^{\prime \prime}$ $3^{\prime} 0^{\prime \prime} \times 7^{\prime} 0^{\prime \prime}$ $26^{\prime \prime} \times 26^{\prime \prime}$ $26^{\prime \prime} \times 26^{\prime \prime}$

Beads for glass included.

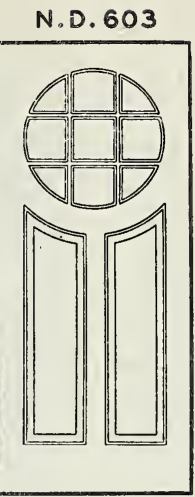

Stiles and top rail Cross rail top rail $-10-0.078^{\prime \prime}$ Mullion ............ $458^{\prime \prime}$ Bottom rail _. . .

Raised panels 2 sides. Can also be furnished with 3-ply plywood flat panels, if desired. Sticking: Standard.

Size of door $\quad$ Approx. glass opg.
$2^{\prime} 8^{\prime \prime} \times 6^{\prime} 8^{\prime \prime}$
$3^{\prime} 0^{\prime \prime} \times 6^{\prime} 8^{\prime \prime}-1.20$
(1)

Beads for glass included.

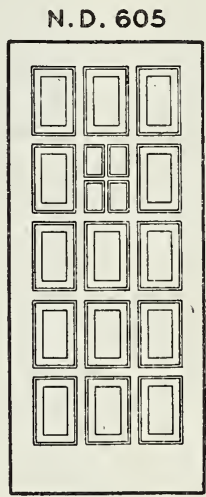

Stiles and top rail . Cross rails and muntins ........... $256^{\prime \prime}$ Bottom rail... . .

Raised panels 2 sides. Can also be furnished with 3-ply plywood flat panels, if desired. Sticking: Standard.

Size of door

Approx. glass opg. $2^{\prime} 8^{\prime \prime} \times 6^{\prime} 8^{\prime \prime}$ $3^{\prime} 0^{\prime \prime} \times 7^{\prime} 0^{\prime \prime}$

Beads for glass included. 


\section{EXTERIOR DOORS-Continued}

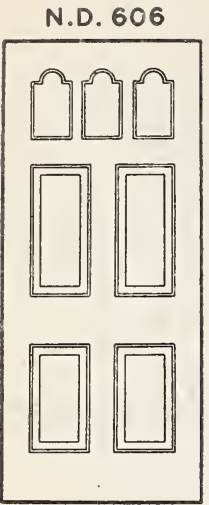

Stiles and top rail

$51 / 21$ Top eross rail and mullo........... 8 " or $93 / 4^{\prime \prime}$

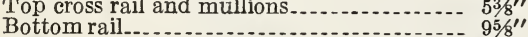

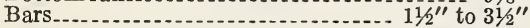

Raised panels 2 sides. Sticking: Standard.

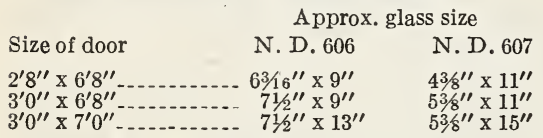

Beads for glass included.

N.D. 609

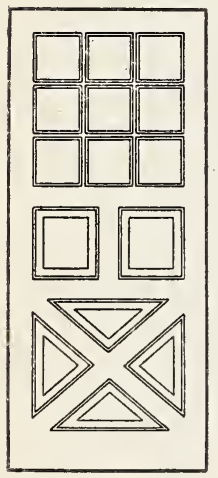

Stiles and top rail Lock rail top rail. $-0-0.4 \%{ }^{\prime \prime}$ Cross rail and mullions. Bottom rail...............

Hip raised panels 2 sides. ( $13 / 8^{\prime \prime}$ or $134^{\prime \prime}$.) Sticking: Standard.

\section{Approx. glass size}

\begin{tabular}{|c|c|c|}
\hline Size of door & N. D. 609 & N. D. 610 \\
\hline $\begin{array}{l}2^{\prime} 8^{\prime \prime} \times 6^{\prime} 8^{\prime \prime} \\
3^{\prime} 0^{\prime \prime} \times 6^{\prime} 8^{\prime \prime} \\
3^{\prime} 0^{\prime \prime} \times 7^{\prime} 0^{\prime \prime}\end{array}$ & $\begin{array}{r}71 / 2^{\prime \prime} \times 75 / 8^{\prime \prime} \\
813 / 16^{\prime \prime} \times 75 / 8^{\prime \prime} \\
8^{13} / 16^{\prime \prime} \times 9^{\prime \prime}\end{array}$ & $\begin{array}{l}55 / 8^{\prime \prime} \times 75 / 8^{\prime \prime} \\
65 / 8^{\prime \prime} \times 75 / 8^{\prime \prime} \\
65 / 8^{\prime \prime} \times 9^{\prime \prime}\end{array}$ \\
\hline
\end{tabular}

Beads for glass included.
N.D. 608

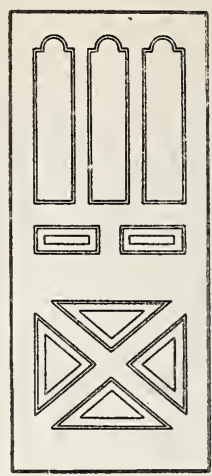

Stiles and top rail.

Cross rail and mullions ---1

Bottom rail.......... Bars -...

Heavy raised panels 2 sides. ( $\left(3 / 4^{\prime \prime}\right.$ or $11 / 8^{\prime \prime}$.) Sticking: Standard.

Size of door

Approx. glass size

$2^{\prime} 8^{\prime \prime} \times 6^{\prime} 8^{\prime \prime}$

$3^{\prime} 0^{\prime \prime} \times 6^{\prime} 8^{\prime \prime}$

Approx. glass size $3^{\prime} 0^{\prime \prime} \times 7^{\prime} 0^{\prime \prime}$

$73 / 8^{\prime \prime} \dot{x} 267 / 8^{\prime \prime}$ $73 / 8^{\prime \prime} \times 307 / 8^{\prime \prime}$

Beads for glass included.
N.D. 612

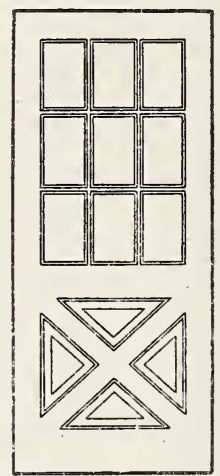

N.D. 613

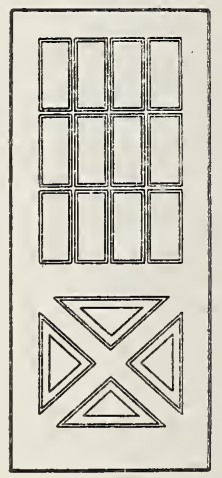

Stiles and top rail.

Lock rail

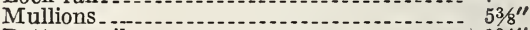
Bottom rail.......... $83 / 4^{\prime \prime}$

Heavy raised panels 2 sides. $\left(3 / 4^{\prime \prime}\right.$ or $11 / 8^{\prime \prime}$.) Sticking: Standard.

\section{Approx. glass size}

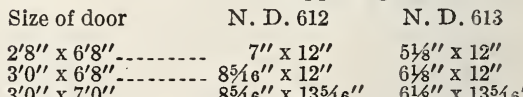

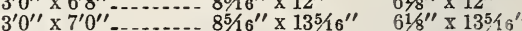
Beads for glass included. 
EXTERIOR DOORS-Continued
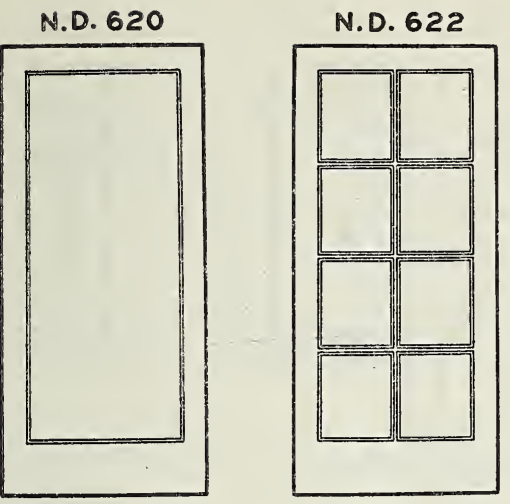

N.D. 623

N.D. 624
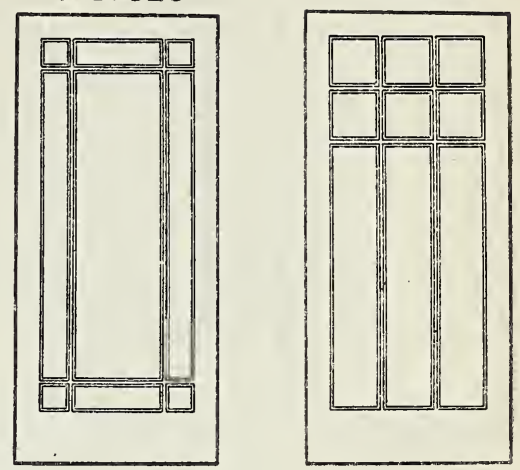

N.D. 625

N.D. 626

N.D. 627
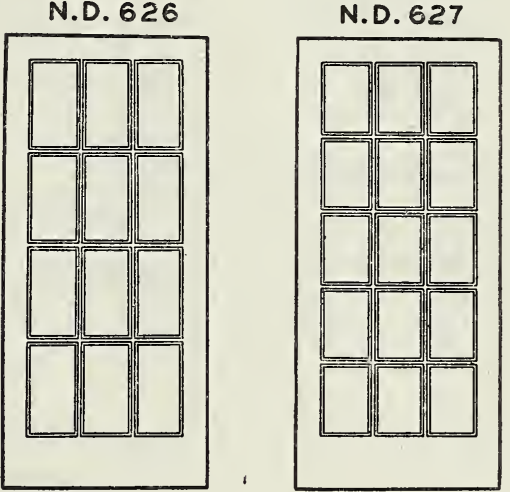

N.D. 630

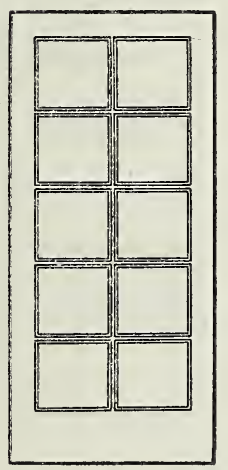

Stiles and top rail

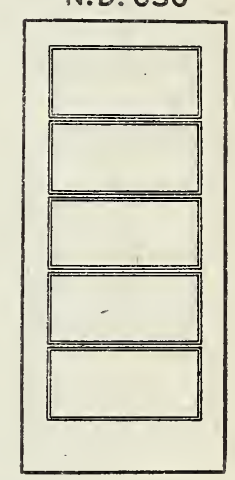

Bottom rail

Sticking: Standard.

Door No.

N. D. 620

N. D. 622

N. D. 623

N. D. 624

N. D. 625

N. D. 626

N. D. 630
Approximate glass size

\begin{tabular}{|c|c|c|c|}
\hline \multicolumn{4}{|c|}{ Approximate glass size } \\
\hline $\begin{array}{l}\text { Size of door } \\
2^{\prime} 0^{\prime \prime} \times 6^{\prime} 8^{\prime \prime}\end{array}$ & $\begin{array}{l}\text { Size of door } \\
2^{\prime} 6^{\prime \prime} \times 6^{\prime} 8^{\prime \prime}\end{array}$ & $\begin{array}{l}\text { Size of door } \\
2^{\prime} 8^{\prime \prime} \times 6^{\prime} 8^{\prime \prime}\end{array}$ & $\begin{array}{l}\text { Size of door } \\
3^{\prime} 0^{\prime \prime} \times 6^{\prime} 8^{\prime \prime}\end{array}$ \\
\hline 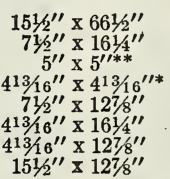 & $\begin{array}{c}211 / 2^{\prime \prime} \times 6616^{\prime \prime} \\
101 / 2^{\prime \prime} \times 1614^{\prime \prime} \\
5^{\prime \prime} \times 5^{\prime \prime * *} \\
613 / 16^{\prime \prime} \times 66^{13} / 16^{\prime \prime *} \\
101 / 2^{\prime \prime} \times 128^{\prime \prime} \\
6^{13} / 16^{\prime \prime} \times 1614^{\prime \prime} \\
613 / 6^{\prime \prime} \times 1278^{\prime \prime} \\
211 / 2^{\prime \prime} \times 1278^{\prime \prime}\end{array}$ & 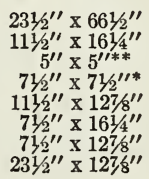 & 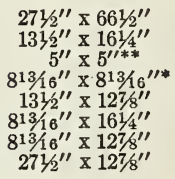 \\
\hline
\end{tabular}

Beads for glass included.

${ }^{*}$ Corner Lights. ${ }^{*}$ Top Lights. 
EXTERIOR DOORS-Continued
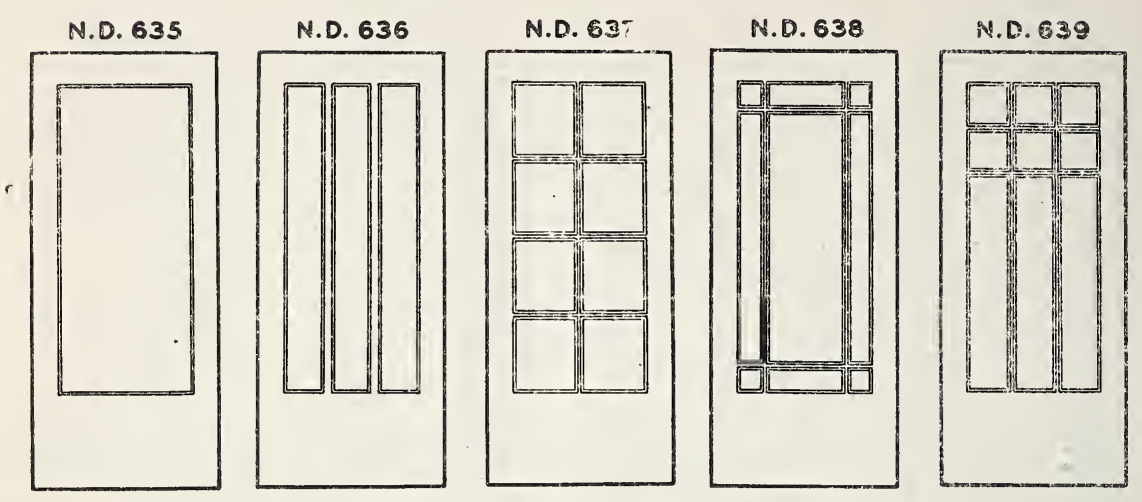

N.D. 640

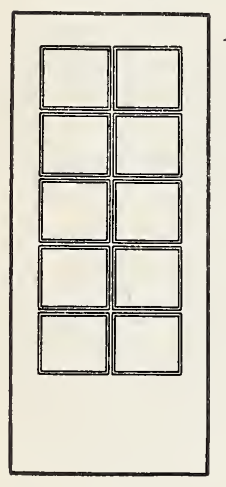

Stiles

Top rail .

Bottom rail
N.D. 641

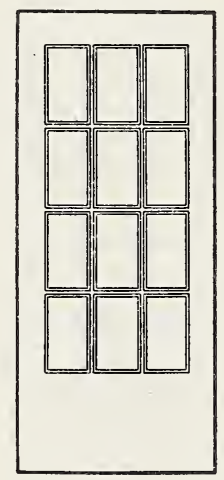

N.D. 642

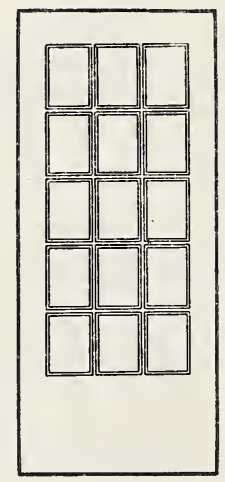

N.D. 644

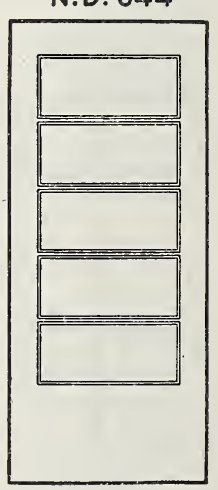

$3^{\prime} 0^{\prime \prime}$ wide and Over $3^{\prime} 0^{\prime \prime}$ under

$512^{\prime \prime}$
$61 / 2 "$

$1812^{\prime \prime}$

wide

$612^{\prime \prime}$

$1812^{\prime \prime}$

Sticking: Standard.

Approximate glass size (for doors with $51 / 2^{\prime \prime}$ stiles)

Door No.

N. D. 635

N. D. 636

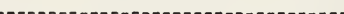

N. D. 637

N. D. 638

N. D. 639

N. D. 640

N. D. 641

N. D. 642

N. D. 644

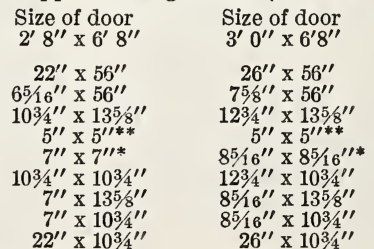

Size of door $3^{\prime} 0^{\prime \prime} \times 7^{\prime} 0^{\prime \prime}$ $26^{\prime \prime} \times 60^{\prime \prime}$ $12^{3} 4^{\prime \prime} \times 145 / 8^{\prime \prime}$ $1234^{\prime \prime} \times 14 \%$ $85 / 16^{\prime \prime} \times 85 / 16^{\prime \prime *}$ $12^{3} 4^{\prime \prime} \times 11 \%$ " $6^{\prime \prime}$ $85 / 16^{\prime \prime} \times 145 / 8^{\prime \prime}$ $85 / 16^{\prime \prime} \times 11 \% 16^{\prime \prime}$ $26^{\prime \prime} \times 11 \% 16^{\prime \prime}$

Beads for glass included.

**Corner Lights. ${ }^{*}$ Top Lights. 


\section{SIDE LIGHTS}

\section{S.L. 675}

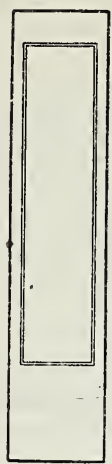

Stiles

Top rail.

Bottom rail doors with which they are used.

Sticking: Standard.

Beads for glass included.

\section{S.L. 676}

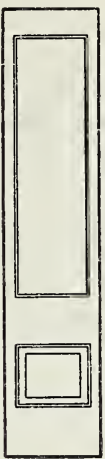

Stiles Top rail Lock rail..... $8^{\prime \prime}$ or $95 / 8^{\prime \prime}$ wide

Top and bottom rails made same width as in doors with which they are used.

Sticking: Standard.

Beads for glass included.

\section{STORM DOORS}

N.D. 702

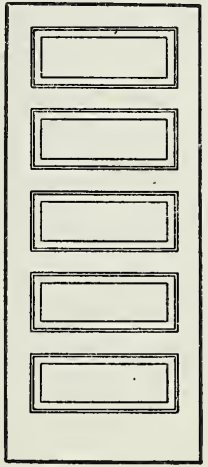

Stiles and top rail

Cross rail.

Bottom rail 2 sides. Can also be furnished plywood flat panels, if desired.

Sticking: Standard.

$$
\begin{aligned}
& \text { Size of door } \\
& 2^{\prime} 6^{\prime \prime} \times 6^{\prime} 7^{\prime \prime} \\
& 2^{\prime} 8^{\prime \prime} \times 6^{\prime} 9^{\prime \prime} \\
& 2^{\prime} 10^{\prime \prime} \times 6^{\prime} 11^{\prime \prime} \\
& 3^{\prime} 0^{\prime \prime} \times 7^{\prime} 1^{\prime \prime}
\end{aligned}
$$

\section{N.D. 703}

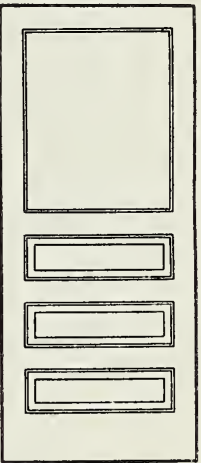

Stiles and top rail_._._. Cross rail. Bottom rail.

Raised panels 2 sides. Can also be furnished with 3-ply plywood flat panels, if desired. Sticking: Standard.

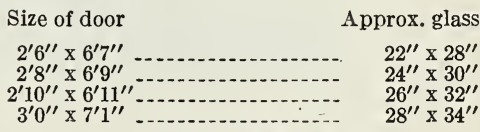

Beads for glass included. 


\section{CUPBOARD DOORS}

N.D. 710

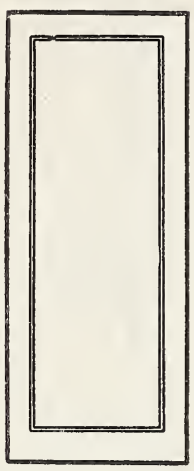

Stiles and top
Bottom rail.

$$
214^{\prime \prime} \text { or } 312^{\prime \prime}
$$
$314^{\prime \prime}$ or $4 \frac{1}{2^{\prime \prime}}$

3-ply plywood flat panel. Sticking: Standard. Can also be furnished solid raised panel one side, flat one side.

\section{N.D. 711}

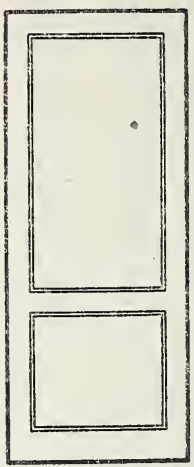

Stiles, top \& cross rail. . . . . . 2 21/4" or $^{-1} 312^{\prime \prime}$ Bottom rail. . . .

3-ply plywood flat panels. Sticking: Standard. Can also be furnished solid raised panel one side flat one side.

\section{N.D. 712}

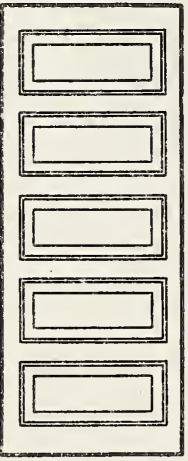

Stiles, top \& cross rail . . . ..... 21/4" or $31 / 2^{\prime \prime}$ Bottom rail.

Raised panels 2 sides. Sticking: Standard. Can also be furnished 3-ply plywood flat panels, if desired.

N. D. 712 doors are made as follows:

Doors up to and including $2^{\prime} 0^{\prime \prime}$ high

2 cross panels.

Over $2^{\prime} 0^{\prime \prime}$ up to and including $3^{\prime} 0^{\prime \prime}$ high....... 3 cross panels.

Over $3^{\prime} 0^{\prime \prime}$ up to and including $4^{\prime} 0^{\prime \prime}$ high

Over $4^{\prime} 0^{\prime \prime}$ high................................. 5 cross panels. 


\section{GARAGE DOORS}

N.D. 720

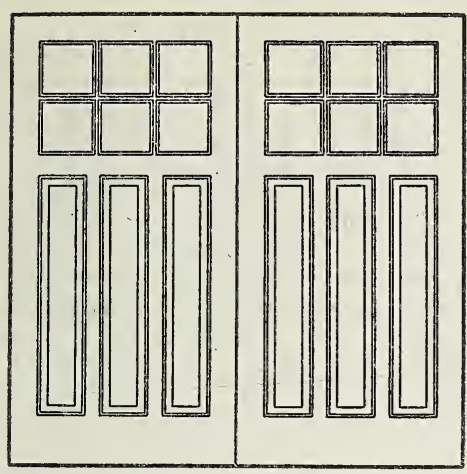

Stiles and top rail . . . . .

Cross rail Mullions _....... $\begin{array}{ll}\text { Bottom rail } & \\ \text { Vertical and horizontal bars between glass.- } & 95 / 8^{\prime \prime}\end{array}$

Raised panels 2 sides. Can also be furnished with 3-ply plywood flat panels, if desired. Sticking: Standard.

Beads for glass included.

N.D. 722

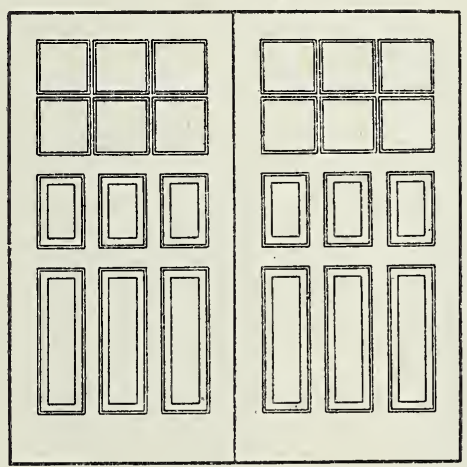

Stiles and top rail ..................... $51 / 2^{\prime \prime}$ Cross rail_._. Mullions ....................... $37 / 8^{\prime \prime}$

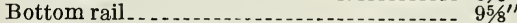
Vertical and horizontal bars between glass.- $1 / 2{ }^{\prime \prime}$

Raised panels 2 sides. Can also be furnished with 3-ply plywood flat panels, if desired. Sticking: Standard.

Beads for glass included.
N.D. 721

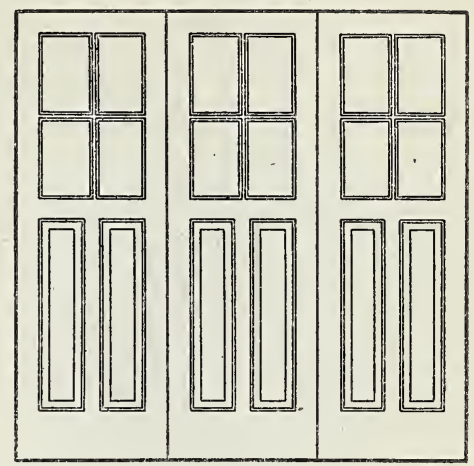

Stiles and top rail .............. $434^{\prime \prime}$

Cross rail_........... $45 / 8^{\prime \prime}$ Mullions . . . . Bottom rail _. .

Vertical and horizontal bars between glass.- $1 / 2{ }^{\prime \prime}$

Raised panels. Can also be furnished with 3-ply plywood flat panels, if desired. Sticking: Standard.

Beads for glass included.

N.2. 723

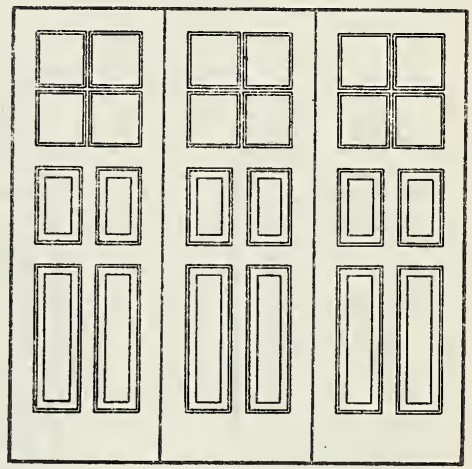

Stiles and top rail ....................... $434^{\prime \prime}$ Cross rail_..._...................... $458^{\prime \prime}$ Mullions _............................... 37,8', Bottom rail. Vertical and horizontal bars between glass.- $1 / 2{ }^{\prime \prime}$

Raised panels 2 sides, Can also be furmished with 3-ply plywood flat panels, if desired. Sticking: Standard.

Beads for glass included. 


\section{GARAGE DOORS-Continued}

N.D. 724

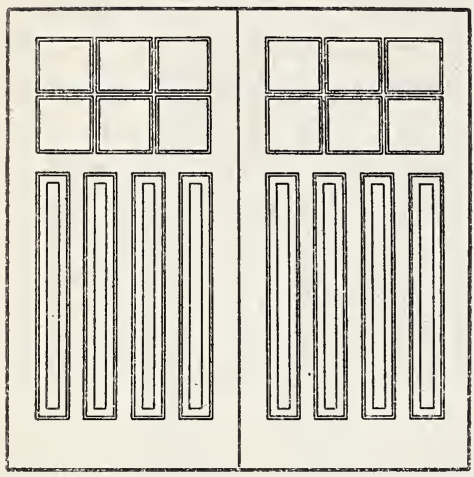

Stiles and top rail _.

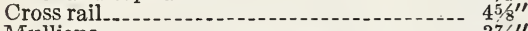
Mullions . Bottom rail

Raised panels 2 sides. Can also be furnished with 3-ply plywood flat panels, if desired. Sticking: Standard.

Beads for glass included.
N.D. 725

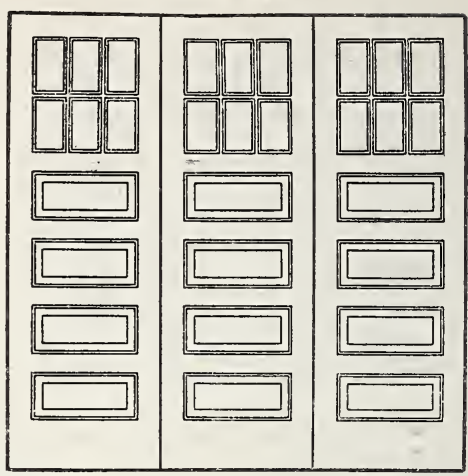

Stiles and top rail Cross rail Bottom rail

Vertical and horizontal bars between glass.- 1/2"

Raised panels 2 sides. Can also be furnished with 3-ply plywood flat panels, if desired. Sticking: Standard.

Beads for glass included.

TOILET DOORS

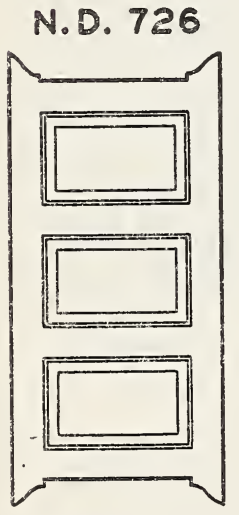

Stiles and top rail. ........ $434^{\prime \prime}$ Cross rails._.

Raised panels 2 sides, Stick. ing: Standard.

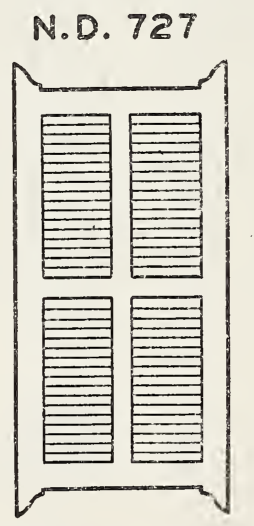

Stiles and top rail _....... $31 / 2^{\prime \prime}$ Cross rails and mullions... $23 \% 8^{\prime \prime}$

Stationary slats. Sticking: Square.

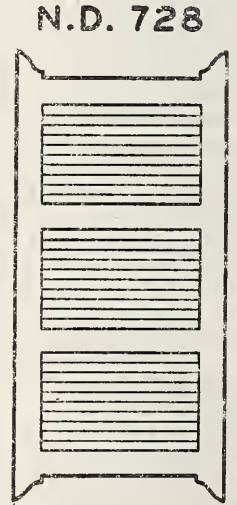

Stiles and top rail_._._... $31 / 2$ " Cross rails.............. $33 / 8^{\prime \prime}$

Stationary slats. Sticking: Square.

Toilet doors can be supplied without lugs, if desired. 
BLIND OR SUMMER DOORS

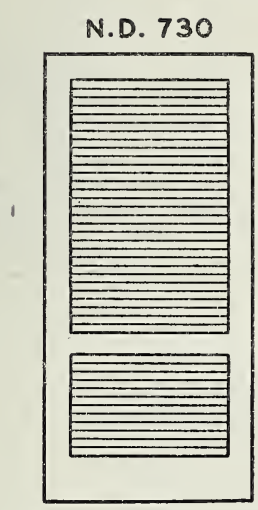

Stiles and top rail

Cross rail

Bottom rail

Stationary slats. Sticking: Square.
M. D. 3 !

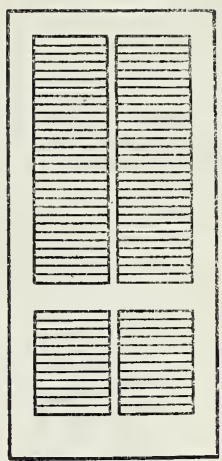

Stiles and top rail _.........

Cross rail

Mullions ${ }_{\text {Bil }}$

Stationary slats. Sticking: Square.

\section{COMBINATION DOORS}

N.D. 735

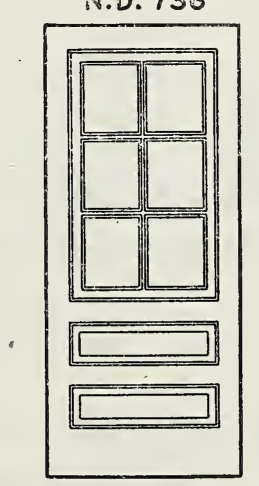

Stiles, top and cross rails . . . . . . . $414^{\prime \prime}$ to $434^{\prime \prime}$ Bottom rail........................ $9^{\prime \prime}$ or $958^{\prime \prime}$ 
COMBINATION DOORS-Continued

N.D. 737

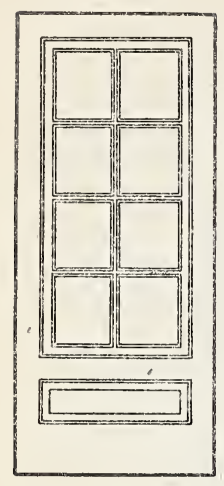

N.D. 739

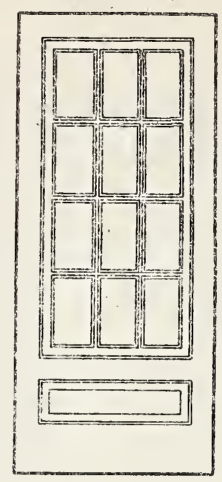

N.D. 742

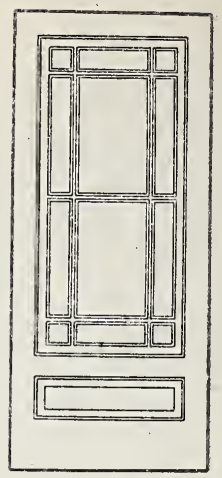

N.D. 749

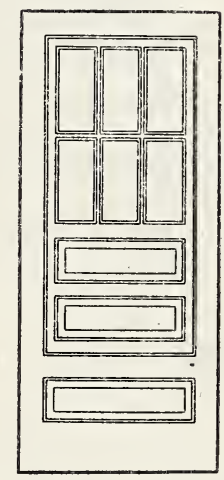

Stiles, top and cross rails Bottom rail.
N.D. 756

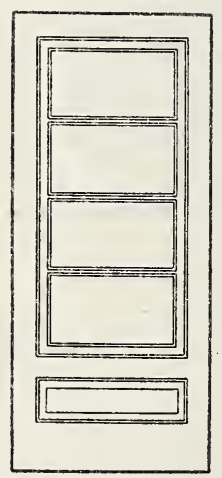

$414 "$ to $434^{\prime \prime}$

\section{PONDEROSA PINE FLUSH DOORS}

25. All commercial standard ponderosa pine flush doors shall meet the following requirements:

26. Material.-Ponderosa pine used in the manufacture of flush doors shall be properly kiln-dried. A water-resistant glue shall be used.

27. Workmanship.-Flush doors shall be well manufactured and flat surfaces smoothly machine-sanded.

28. Construction.- The core of flush doors shall be formed of vertical blocks not over 2 inches wide on the face, securely glued together with water-resistant glue and with joints well staggered and the core surrounded with $3 / 4$-inch edge strip on all four edges. In lieu of vertical blocks, the core may be of stile, rail, and panel units, each unit made up of blocks, all of which when assembled with dowels, will make a solid core foundation for the cross banding and veneer. Cores shall be uniformly dried to proper moisture content and dressed to a smooth. 
surface before applying the crossbanding. If crossbanded, the crossbanding and face veneers shall be glued to each side of the core and assembled under pressure. Exterior flush doors may be manufactured with wide edge strips to allow cutting down width and height of doors and they may also be made to permit cutting circle or other irregular top.

29. Veneers.-If cross-banded, the crossbanding shall be not less than $1 / 16$ inch or more than $1 / 8$ inch thick. Face veneers shall be from $1 / 16$ inch to $1 / 4$ inch thick before sanding, except where $V$-grooving is required, then $1 / 4$ inch thick before sanding.

30. Thickness.-Flush doors shall be $1 \frac{3}{4}$ inches thick and a thickness tolerance of minus $1 / 16$ inch shall be allowed.

31. Grading.-Flush doors shall be of No. 1 grade. The stock shall be clear, except that bright sap, light-brown stain, and light-red kiln burn shall be permitted.

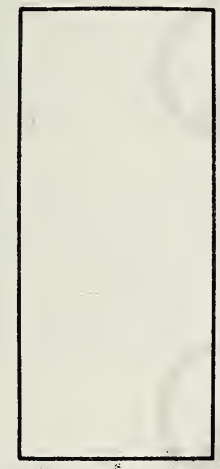

PLAIN FLUSH

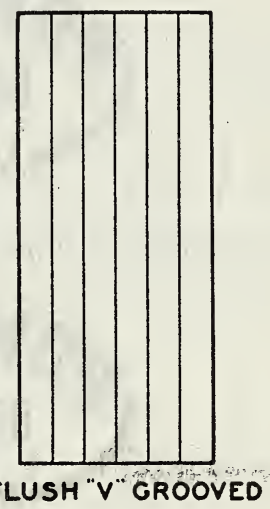

FLUSH "V "GROOOED

Light openings may be cut in these doors to suit the wishes of the purchaser.

\section{INSPECTION}

32. All ponderosa pine doors sold as conforming to the commercial standard are subject to inspection in the condition received, and complaints regarding any shipment shall be made within ten (10) days after receipt thereof. Any rejected doors shall be held, properly protected, for a period of thirty (30) days after notice of rejection and pending adjustment.

\section{LABELING}

33. In order to assure the purchaser that he is getting ponderosa pine doors of the quality specified, producers may individually, or in concert with their trade associations, issue guarantees, or grade mark each door by stamp, brand, or label as conforming to this standard. The following wording is recommended for the label:

This GRADE _...... ponderosa pine door is guaranteed by the manufacturer to conform to Commercial Standard CS120-44, as issued by the National Bureau of Standards of the U. S. Department of Commerce. 


\section{Grade Marking.}

34a. The following grade-marking rules have been adopted by the National Door Manufacturers Association, Inc., as a means of assuring consumers and distributors that ponderosa pine doors conform to the high standards of quality defined herein.

$34 \mathrm{~b}$. Consumers and distributors may request that ponderosa pine doors be grade-marked. All ponderosa pine doors guaranteed to conform to the commercial grade rules as set forth herein may be stamped, labeled, or branded with the letters "NDMA", the grade designation, and an identification of the manufacturer by numerals.

34c. The following official grade designations have been approved by the National Door Manufacturers Association:

(1) For ponderosa pine doors of No. 1 grade:

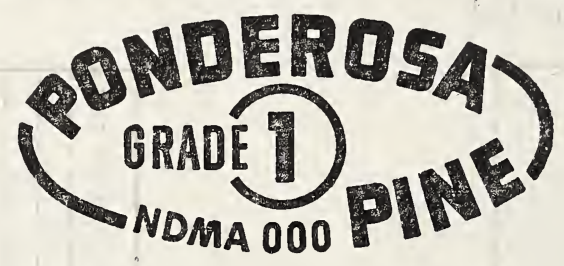

(2) For ponderosa pine doors of No. 2 grade:

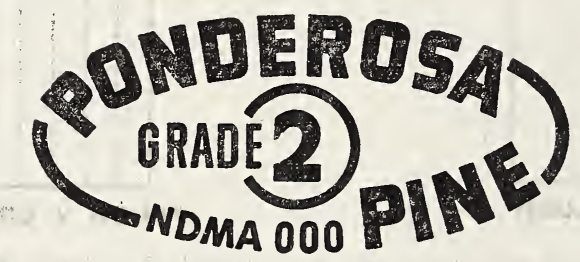

(3) For ponderosa pine doors of "Millrun" grade:

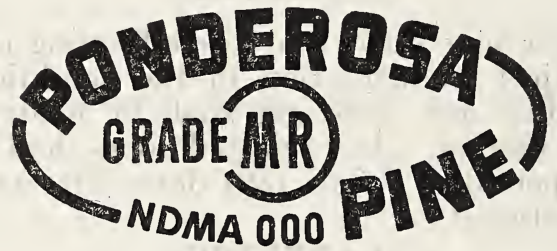

\section{NOMENCLATURE AND DEFINITIONS}

The definitions below give the meaning of various terms used in this standard:

Bars.-Wood divisions separating lights of glass.

Coped.-The shaping of the ends of rails, mullions, muntins, or bars so that they will cover and fit the contour of the sticking.

Core.-The innermost layer in veneered door construction.

Crossbanding.-The veneer which may be used in the construction of flush doors, which is placed between the core and face veneers with the direction of the grain at right angles to that of the face veneer. 
Flush door.-Made up of a core, crossbanding, and face veneers, or core and face veneers only.

Panel door.-Made up of stiles, rails, and one or more panels, the stiles and rails forming the frame around the panel.

Sash door.-Same as panel door, except one or more panels are replaced by glass.

Kiln dried.-Dried in a closed chamber in which the removal of moisture is controlled by artificial heat and usually by relative humidity.

Mullion.-An upright or vertical, bar in a door.

Muntin.-Any short or light bar, either vertical or horizontal, in a door between glass or panels and not extending the full width or length of the door.

Plywood panel. - A panel made up of core and face veneer.

Pitch seam.-An opening or imperfection parallel to the grain which is filled with pitch.

Rails.- The cross, or horizontal, pieces of the framework of a door. Bottom rail.-The bottom cross, or horizontal, piece of a door.

Lock rail. - The wide cross, or horizontal, rail of a door at lock height.

Top rail.-The top cross, or horizontal, piece of a door.

Sticking.-A mold which is worked on the edges of stiles, rails, mullions, muntins, or bars, adjacent to panels or glass.

Stiles.-The upright, or vertical, outside pieces of a door.

Veneered.-Made up of core and face veneers (may include crossbanding in flush doors).

\section{USE CLASSIFICATION INDEX}

INTERIOR DoORS

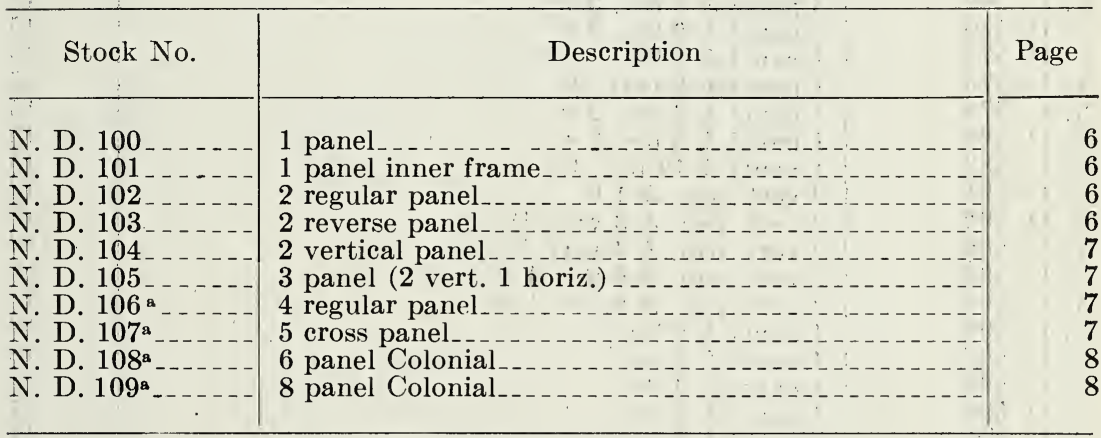

EXTERIOR Doors

N. D. 110

N. D. 112

N. D. 500

N. D. 501

N. D. 502

N. D. 505

N. D. 506

N. D. 507

N. D. 508

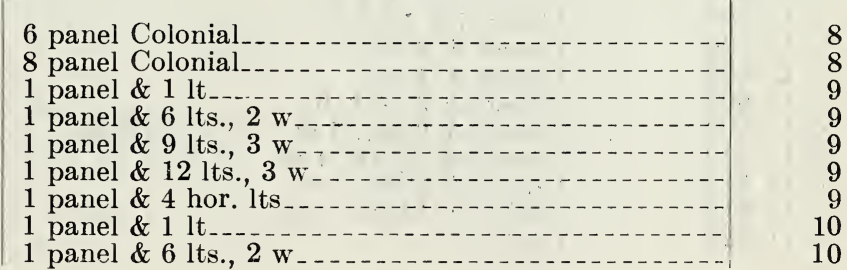

a Also for exterior use. 
Exterior Doors-Continued

\begin{tabular}{|c|c|c|}
\hline Stock No. & Description & Page \\
\hline N. D. 509 & 1 panel \& 9 lts., $3 \mathrm{w}_{-}$ & 10 \\
\hline N. D. $510_{-}$ & 1 panel \& 8 lts., 2 w & 10 \\
\hline N. D. 511 & 1 panel \& 9 marg. 1 ts & 10 \\
\hline N. D. 512 & 1 panel \& 12 lts., $3 \mathrm{w}$ & 10 \\
\hline N. D. 513 & & 11 \\
\hline $\begin{array}{l}\text { N. D. } 514 \\
\text { N. D. } 515\end{array}$ & $\begin{array}{l}2 \text { hor. pan. \& } 1 \text { ltt. } \\
2 \text { hor. pan. \& } 4 \text { lts., } 2 \mathrm{w}_{-}\end{array}$ & 11 \\
\hline N. D. 516 & 2 hor. pan. \& 6 lts., $3 \mathrm{w}_{-}$ & $\begin{array}{l}11 \\
11\end{array}$ \\
\hline N. D. 5 & 2 hor. pan. \& 9 lts., 3 w & 11 \\
\hline N. D. 5 & 2 hor. pan. \& 3 vert. 1 ts & 11 \\
\hline N. D. 5 & 2 hor. pan. \& 12 lts., 3 w & 11 \\
\hline N. D. 5 & & 12 \\
\hline $\begin{array}{l}\text { N. D. } 530- \\
\text { N. D. } 531_{-}\end{array}$ & $\begin{array}{l}3 \text { hor. pan. \& } 1 \text { lt. } \\
3 \text { hor. pan. \& } 4 \text { lts., } 2 \mathrm{w}_{-}\end{array}$ & 12 \\
\hline N. D. 532 & $\begin{array}{l}\text { n. \& } 4 \text { lts., } 2 \text { w.- } \\
\text { n. \& } 6 \text { lts., } 3 \text { w.. }\end{array}$ & $\begin{array}{l}12 \\
12\end{array}$ \\
\hline N.D. 533 & 3 hor. pan. \& 3 vert. lts & 12 \\
\hline N. D. 5 & 3 hor. pan. \& 3 hor. 1 ts & 13 \\
\hline N. D. 5 & 3 hor. pan. \& 1 lt & 13 \\
\hline N. D. 5 & 3 hor. pan. \& 4 lts., $2 \mathrm{w}_{-}$ & 13 \\
\hline $\begin{array}{l}\text { N. D. } 5 \\
\text { N. D. }\end{array}$ & $\begin{array}{l}3 \text { hor. pan. } \\
3 \text { hor. pan. }\end{array}$ & 13 \\
\hline $\begin{array}{l}\text { N. D. } 5 \\
\text { N. D. } 5\end{array}$ & $\begin{array}{l}3 \text { hor. pan. \& } 9 \text { lts., } 3 \mathrm{w}_{-} \\
3 \text { hor. pan. \& } 3 \text { vert. lts }\end{array}$ & $\begin{array}{l}13 \\
13\end{array}$ \\
\hline N. D. 5 & 5 panel \& 1 it & 14 \\
\hline N.D. 5 & 4 hor pan. \& 1 lt & 14 \\
\hline N. D. 5 & 2 vert. pan. \& $1 \mathrm{lt} \ldots$ & 14 \\
\hline N. D. & n. \& 4 lts., 2 w - & 14 \\
\hline N. D. 5 & , $2 \mathrm{w}_{-} \ldots$ & 14 \\
\hline N.D. 5 & s., $3 \mathrm{w}_{-} \ldots$ & 14 \\
\hline $\begin{array}{l}\text { N. D. } \\
\text { N. D. }\end{array}$ & 2 vert. pan. \& 3 hor. 1 ts & 14 \\
\hline $\begin{array}{l}\text { N. D. } 5 \\
\text { N. D. } 5\end{array}$ & $2 w_{-}$ & 15 \\
\hline N. D. 5 & $\begin{array}{l}3 \text { panel \& } 4 \text { its., } 2 \mathrm{w} \\
3 \text { panel } \& 6 \text { its., } 3 \text { w }\end{array}$ & 15 \\
\hline N. D. & $\& 9$ lts., $3 \mathrm{w}$ & 15 \\
\hline N. D. 5 & el \& 1 lt $\ldots \ldots$ & 15 \\
\hline N. D. 5 & 1 panel \& 3 & 15 \\
\hline N. D. 5 & 1 panel \& 6 lts., 2 w $\ldots$ & 15 \\
\hline N. D. 5 & \& 9 lts., 3 w $\ldots$ & 15 \\
\hline $\begin{array}{l}\text { N. D. } \\
\text { N. D. }\end{array}$ & 1 panel \& 12 lts., $3 \mathrm{w}_{\ldots} \ldots$ & 15 \\
\hline $\begin{array}{l}\text { N. D. } \\
\text { N. D. }\end{array}$ & $\begin{array}{l}\text { pan. \& } 1 \text { ltt. } \\
\text { pan. \& } 3 \text { vert. lts }\end{array}$ & $\begin{array}{l}16 \\
16\end{array}$ \\
\hline N. D. & $\begin{array}{l}\text { \& } 3 \text { vert. } 1 \text { tis } \\
\& 4 \text { vert. } 1 \text { ts }\end{array}$ & $\begin{array}{l}16 \\
16\end{array}$ \\
\hline N.D. & 2 vert. pan. \& 6 lts., $3 \mathrm{w}_{-}$ & 16 \\
\hline & 2 vert. pan. \& 8 lts., $4 \mathrm{w}_{-}-$ & 16 \\
\hline N. D. & 4 panel \& 21 & 16 \\
\hline N D & $1 \& 31$ & 16 \\
\hline N. I & $-\ldots \ldots-\ldots$ & 16 \\
\hline N. D & $4 \mathrm{p}$ & 17 \\
\hline $\begin{array}{l}\text { N. D } \\
\text { N. D }\end{array}$ & 4 panel \& $8 \mathrm{lts}_{2} \ldots$ & 17 \\
\hline D. & $\begin{array}{l}2 \text { vert. pan. \& } 9 \mathrm{lts} \ldots \\
2 \text { vert. pan. \& } 7 \mathrm{lts}_{\ldots} \ldots\end{array}$ & $\begin{array}{l}17 \\
17\end{array}$ \\
\hline N. & $1 \& 4$ lts $\ldots$ & 17 \\
\hline & $\& 3 \mathrm{lts}_{-}$ & 18 \\
\hline & & 18 \\
\hline N. D & $\operatorname{lts}_{-}$ & 18 \\
\hline N. I & 6 panel \& 9 lts., $3 \mathrm{w}_{-}-$ & 18 \\
\hline N. & $\begin{array}{l}6 \text { panel \& } 12 \text { lts., } 4 \mathrm{w}_{-} \\
4 \text { panel \& } 9 \text { lts., } 3 \mathrm{w}_{-}\end{array}$ & 18 \\
\hline N. D. & 4 panel \& 12 lts., $4 \mathrm{w}$ & 18 \\
\hline N. D. 620 & 1 light $\ldots \ldots$ & 19 \\
\hline
\end{tabular}


Exterior Doors-Continued

\begin{tabular}{|c|c|c|}
\hline Stock No. & Description & Page \\
\hline N. D: $622^{b}$ & 8 lights, $2 w_{\ldots}$ & 19 \\
\hline N. D. $623^{b}$ & 9 marg. $1 \mathrm{ts}$ & 19 \\
\hline N. D. $624^{\mathrm{b}}$ & 9 lights, $3 \mathrm{w}_{-}$ & 19 \\
\hline N. D. $625^{b}$ & 10 lights, $2 \mathrm{w}_{\text {- }}$ & 19 \\
\hline N. D. $626^{\text {b }}$ & 12 lights, $3 w_{-}$ & 19 \\
\hline N. D. $627^{\text {b }}$ & 15 lights, $3 \mathrm{w}_{\ldots}$ & 19 \\
\hline N. D. $630_{-}$ & 5 hor. lights & 19 \\
\hline N. D. 635 & 1 light & 20 \\
\hline N. D. 636 & 3 vert. lights_- & 20 \\
\hline N. D. $637_{-}$ & 8 lights, 2 w $\ldots$ & 20 \\
\hline N. D. 638 & 9 marg. lights & 20 \\
\hline N. D. 6 & 9 lights, 3 w..... & 20 \\
\hline N. D. 640 & 10 lights, $2 w_{-}$ & 20 \\
\hline N. D. 641 & 12 lights, $3 \mathrm{w}_{\ldots} \ldots$ & 20 \\
\hline N. D. 642 & 15 lights, $3 \mathrm{w} \ldots$ & 20 \\
\hline & 5 nor & \\
\hline
\end{tabular}

\section{Sidelights}

S. L. 675

N. D. $702 \ldots$
N. D. $703 \ldots$

\section{Cupboard Doors}

\begin{tabular}{l|l|l|l}
\hline N. D. $710 \ldots$ & panel & \\
N. D. 711 & 22 \\
N. D. $712 \ldots$ & hanel
\end{tabular}

Garage Doors

N. D. 720
N. D. 721
N. D. 722
N. D. 723
N. D. 724
N. D. 725

3 vert. pan. \& 6 lts., 3 w 2 vert. pan. \& 4 lts., 2 w 6 pan. \& 6 its., $3 \mathrm{w} \ldots$ 4 pan. \& 4 lts., $2 \mathrm{w} \ldots \ldots$ 4 vert. pan. \& 6 lts., 3 w 4 hor. pan. \& 6 lts., 3 w

Tollet Doors

N. D. 726

N. D. 727

N. D. 728
3 hor. panel-

4 stat. slat panel 24 3 stat. slat panel. 
Blind or Summer Doors

\begin{tabular}{l|l|r}
\hline Stock No & Description & Page \\
\hline N. D. $730 \ldots$ & $\begin{array}{l}\text { 2 stat. slat panel_ slat panel } \\
\text { N. D. } 731 \ldots\end{array}$ & 25 \\
\hline
\end{tabular}

Combination Doors

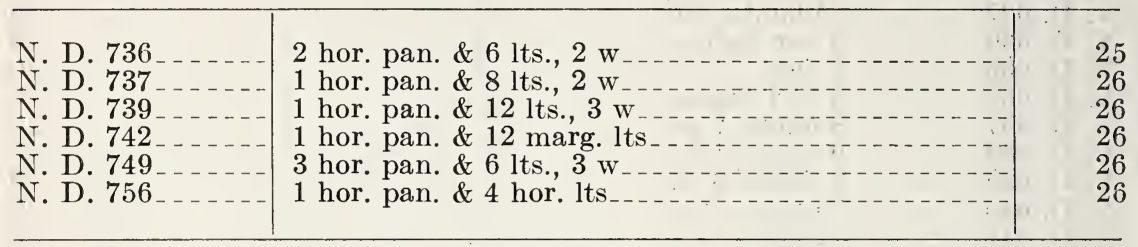

Flush Doors

\begin{tabular}{|c|c|c|}
\hline $\begin{array}{l}\text { Flush } \ldots \ldots \ldots \\
\text { Flush } \ldots \ldots \ldots\end{array}$ & $\begin{array}{l}\text { Plain flush } \\
V \text { grooved }\end{array}$ & $\begin{array}{l}27 \\
27\end{array}$ \\
\hline
\end{tabular}

\section{EFFECTIVE DATE}

35. The standard is effective for new production from September $15,1944$.

\section{STANDING COMMITTEE}

36. The following individuals comprise the membership of the standing committee, which is to review, prior to circulation for acceptance, revisions proposed to keep the standard abreast of progress. Each organization nominated its own representative. Comment concerning the standard and suggestions for revision may be addressed to any member of the committee or to the Division of Trade Standards, National Bureau of Standards, which acts as secretary for the committee:

W. H. Schwa B (chairman), Huttig Manufacturing Co., Muscatine, Iowa.

Grenn Converse, Anson \& Gilkey Co., Merrill, Wis.

Fred A. Hoerner, Farley \& Loetscher Manufacturing Co., Dubuque, Iowa.

R. J. Lillibridge, National Door Manufacturers Association, Inc., 712 Transportation Bldg., Washington, D. C.

Frank Stevens, Ideal Co., Waco, Tex. (Representing Ponderosa Pine. Woodwork).

Claud F. Wilson, Kimball \& Wilson, Inc., 2127 Fenkell Ave., Detroit 3, Mich. (Representing Woodwork Jobbers Service Bureau.)

Norman B. Cove, Hager \& Cove Lumber Co., Lansing, Mich. (Representing Michigan Retail Lumber Dealers Association.)

W. A. Compton, Allen Millwork Manufacturing Co., Shreveport, La. (Representing Southern Sash \& Door Jobbers Association.)

EDWARD A. Poynton, Director of Construction, Office of Indian Affairs, U. S. Department of the Interior, Chicago 54, Ill.

Harold A. Parks, Hardware Manufacturers' Statistical Association, 205 Church St. (P. O. Box 1603), New Haven 6, Conn.

E. W. MACY, Property Standards Unit, Federal Housing Administration, National Housing Agency, Washington 25, D. C. 


\section{HISTORY OF PROJECT}

37. On December 30, 1943, the National Door Manufacturers Association requested the cooperation of the National Bureau of Standards in the establishment of a commercial standard for standard stock ponderosa pine doors. A draft of the proposed standard was submitted on January 29, 1944, to producers, and to a number of technical, distributor, and consumer organizations for their views and comment. All comment was carefully considered at a meeting held in Chicago, Ill., on March 28, 1944. The standard was then adjusted to represent the composite views of all interested groups, and circulated on May 31, 1944, to the trade for written acceptance.

38. Upon receipt of official acceptance, estimated to represent a satisfactory majority of the production by volume, and in the absence of active valid opposition, the standard was promulgated on August 15, 1944, as Commercial Standard CS120-44. 



\section{ACCEPTANCE OF COMMERCIAL STANDARD}

If acceptance has not previously been filed, this sheet properly filled in, signed and returned, will provide for the recording of your organization as an acceptor of this commercial standard.

Division of Trade Standards,

Date

National Bureau of Standards,

Washington, D. C.

Gentlemen:

Having considered the statements on the reverse side of this sheet, we accept the Commercial Standard CS120-44 as our standard of practice in the

Production $^{1} \quad$ Distribution $^{1} \quad$ Use $^{1} \quad$ Inspection $^{1}$

of standard stock ponderosa pine doors.

We will assist in securing its general recognition and use, and will cooperate with the standing committee to effect revisions of the standard when necessary.

Signature of individual officer

(In ink)

(Kindly typewrite or print the following lines)

Name and title of above officer.

Organization

(Fill in exactly as it should be listed)

Street address

City and State

1 Please designate which group you represent by drawing lines through the other three. Please file separate acceptances for all subsidiary companies and affiliates which should be listed separately as acceptors. In the case of related interests, trade papers, colleges, etc., desiring to record their general approval, the words "in principle" should be added after the signature. 


\section{TO THE ACCEPTOR}

The following statements answer the usual questions arising in connection with the acceptance and its significance:

1. Enforcement.-Commercial standards are commodity specifications voluntarily established by mutual consent of those concerned. They present a common basis of understanding between the producer, distributor, and consumer and should not be confused with any plan of governmental regulation or control. The United States Department of Commerce has no regulatory power in the enforcement of their provisions, but since they represent the will of the interested groups as a whole, their provisions through usage soon become established as trade customs, and are made effective through incorporation into sales contracts by means of labels, invoices and the like.

2. The acceptor's responsibility.-The purpose of commercial standards is to establish for specific commodities, nationally recognized grades or consumer criteria and the benefits therefrom will be measurable in direct proportion to their general recognition and actual use. Instances will occur when it may be necessary to deviate from the standard and the signing of an acceptance does not preclude such departures; however, such signature indicates an intention to follow the commercial standard where practicable, in the production, distribution, or consumption of the article in question.

3. The Department's responsibility. - The major function performed by the Department of Commerce in the voluntary establishment of commercial standards on a Nation-wide basis is fourfold: first, to act as an unbiased coordinator to bring all interested parties together for the mutually satisfactory adjustment of trade standards; second, to supply such assistance and advice as past experience with similar programs may suggest; third, to canvass and record the extent of acceptance and adherence to the standard on the part of producers, distributors, and users; and fourth, after acceptance, to publish and promulgate the standard for the information and guidance of buyers and sellers of the commodity.

4. Announcement and promulgation.- When the standard has been endorsed by a satisfactory majority of production or consumption in the absence of active, valid opposition, the success of the project is announced. If, however, in the opinion of the standing committee or the Department of Commerce, the support of any standard is inadequate, the right is reserved to withbold promulgation and publication. 


\section{ACCEPTORS}

39. The organizations and individuals listed below have accepted these grading specifications as their standard of practice in the production, distribution, and use of standard stock ponderosa pine doors. Such endorsement does not signify that they may not find it necessary to deviate from the standard, nor that producers so listed guarantee all of their products in this field to conform with the requirements of this standard. Therefore, specific evidence of quality certification should be obtained where required.

\section{A SSOCIATIONS}

Appalachian Hardwood Manufacturers, Inc., Cıncinnati, Ohio.

Associated General Contractors of America, Inc., The, Washington, D. C.

Building Officials Conference of America, Washington, D. C.

Carolina Lumber \& Builđing Supply Association, Charlotte, N. C. (In principle.)

Cleveland Lumber Institute, The, Cleveland, Ohio. Empire Millwork Association, Buffalo, N. Y.

Hardwood Dimension Manufacturers Association, Louisville, Ky. (In principle.)

Michıgan Retail Lumber Dealers Association, Lansing, Mich.

Mississippı Valley Lumber, Sash \& Door Salesmen's Association, Minneapolis, Minn.

National Door Manufacturers Association, Inc., Chicago, Ill.

New York Lumber Trade Association, New York, N. Y.

Ohio Association of Retall Lumber Dealers, The, Xenla, Ohio.

Ponderosa Pine Woodwork, Chicago, Ill.

Prefabricated Home Manufacturers

Washington, D. C. (In principle.)

Southern California Retail Lumber Association, Angeles, Calif.

Woodwork Jobbers Service Bureau, Chicago, Ill. (In principle.)

\section{FIRMS}

Acme Sash \& Door Co., Cincinnati, Ohio. Adams, Franklin O., Tampa, Fla.

Adams-Rogers Co., Indianapolis, Ina.

Addison-Rudesal Co., Atlanta, Ga.

Adelman Lumber Co., Pittsburgh, $\mathrm{Pa}$.

Adkins \& Co., E. S., Salisbury, Md.

Akron Sash \& Door Co., The, Akron, Ohio.

Allan Lumber Co., Inc., Greencastle, Ind.

Allen Millwork Manufacturıng Co., Shreveport, La. Allith Prouty, Inc., Danville, Ill.

Alto Mills, Inc., Texarkana, Tex.

Amarillo Sash \& Door Co., Houston, Tex.

American Sash \& Door Co., Kansas City, Mo.

Andersen Corporation, Bayport, Minn.

Anderson \& Lind Manufacturing Co., Chicago, Ill.

Andrews, Jones, Biscoe \& Whitmore, Boston, Mass.

Andrews Lumber Co., C. E., New Bethlehem, Pa.

Angel Novelty Co., Fitchburg, Mass.

Angelina County Lumber Co., Keltys, Tex.

Anson \& Gilkey Co., Merrill, Wis.

Arizona Sash Door \& Glass Co., Phoenix, and Tucson, Ariz.

Arkmo Lumber Co., The, Little Rock, Ark.

Armstrong-Thielman Lumber Co., Calumet, Mich.

Asheim, Leonard, Bridgeport, Conn.

Ashton Co., C. J., Detroit, Mich.

A thens Lumber Co., Athens, Ga.

Atkinson-Stutz Co., San Francisco, Calif.

Augusta Lumber Co., Augusta, Ga.

A ves, Inc., Arthur A., Yakima, Wash.

Bach Millwork Co., E. E., Minneapolis, Minn.

Barnes Lumber Co., W. F. \& J. F., Waco, Tex.

Barthmaier, Eugene V., Philadelphia, $\mathrm{Pa}$.

Baxter \& Co., C. B., Kansas City, Mo.

Beasley \& Sons Co., Nashville, Tenn.

Becker Builders Supply Co., The, Wilmington, N. C. Becker-Danowitz Co.., Inc., Brooklyn, N. Y.

Bedard \& Morency Mill Co., Oak Park, Ill.

Belli, Edo J., Chicago, Ill.
Bellman, Gillett \& Richards, Toledo, Ohio.

Bennett-Bailey Lumber Co., Minneapolis, Minn.

Bickford, Robert Turner, Elmira, N. Y.

Binswanger \& Co., Inc., Richmond, $\mathrm{Va}$.

Birmingham Sash \& Door Co., Birmingham, Ala.

Bishop, Horatio W., La Mesa, Calif.

Blithe, Wesley Lesher, Philadelphia, $\mathrm{Pa}$.

Blount Lumber Co., The, Lacona, N. Y.

Boehm, George A., New York, N. Y.

Bogner, Harry, Milwaukee, Wis.

Boise Sash \& Door Factory, Boise, Idaho.

Bommer Spring Hinge Co., Brooklyn, N. Y.

Bosman \& Casson, Inc., Harrison, N. J.

Bovard, William R., Kansas City, Mo. (In principle.)

Brairerd, Harry B., New York, N. Y

Bristol Door \& Lumber Co., Bristol, Va.

Brockway-Smith-Haigh-Lovell Co., Cnarlestown, Mass.

Brown-Borhek Co., Bethlehem, Pa.

Brown Graves Co., Akron, Ohio.

Brust \& Brust, Milwaukee, W' is.

Buckley Door Co., F. S., San Francisco, Calif.

Bucky, Fred W., Jr., Jacksonville, Fla.

Buechner \& Orth, St. Paul, Minn. (In principle.)

Buffalo, City of, Denartment of Public WorksArchitectural Service, Buffalo, N. Y.

Buffelen Lumber \& Manufacturing Co., Fort Worth, Tex., and Detroit, Mich.

Builders Supply Co., B ismarck, N. Dak.

Building Supplies Corporation, Norfolk, Va.

Byron Sash \& Door Co., Inc., Louisville, Ky.

$\mathrm{C} \& \mathrm{M}$ Construction Co., Inc., Philadelphia, Pa. (In principle.)

Calcasieu Lumber Co., Austin, Tex.

California Door Co., The, Los Angeles, Calif.

Cameron \& Co., Inc., Wm., Waco, Tex.

Cameron Lumber Co., Inc., Newburgh, N. Y

Camlet, J. Thomas, Passaic, N. J.

Cannon \& Mullen, Salt Lake City, Utah.

Carder, Macon O., Amarillo, Tex.

Carnahan Manufacturing Co., Loogootee, Ind.

Carr Adams \& Collier Co., Dubuque, Iowa.

Carr \& Johnston Co., Peoria, Ill.

Carroll, John, Asbury Park, N. J.

Carter-Lee Lumber Co., Indianapolis, Ind.

Cavalier Corporation, Chattanooga, Tenn.

Cellar Lumber Co., Westerville, Ohio.

Central Jersey Wholesale Supply Co., Trenton, N. J.

Chapin Lumber Co., The, Denver, Colo.

Charlottesville Lumber Co., Inc., Charlottesville, Va.

Chesler \& Sons Co., J., Brooklyn, N. Y. (In principle.)

Chicago \& Riverdale Lumber Co., Chicago, Ill.

Cincinnati, City of, Cincinnati, ohio.

Cincinnati Sash \& Door Co.. The, Cincinnati, Ohio.

Cleary Millwork Co., Inc., Ansonia, Conn.

Clem Lumber Co., Dallas, Tex.

Cogswell Construction Co., The, Baltimore, Md.

Cole Manufacturing Co., Memphis, Tenn.

Collier-Barnett Co., The, Toledo, Ohio.

Combs Lumber Co., Lexington, $\mathrm{Ky}$.

Connecticut, State of, Purchasing Department, Hartford, Conn.

Conrad \& Cummings, Binghamton, N. Y

Convertible Door Manufacturing Co., Milwaukee, WV is.

Conlbaugh \& Son Co., C. C., Gloucester City, N. J. Coolidgc, Shepley, Bulfinch \& Abbott, Boston, Mass.

Corbin Division, P. \& F., New Britain, Conn. (In principle.) 
Cram \& Ferguson, Boston, Mass.

Cresmer Manufacturing Co., Riverside, Calif. Cross Austin \& Ireland Lumber Co., Brooklyn,

Crowell \& Lancaster, Bangor, Maine.

Curtis Co's., Inc., Clinton, Iowa, Lincoln, Nebr., and other cities.

Curtis Co., Ros, Detroit, Mich.

D'Arcy Co., Dover, N. H.

Davidson Sash \& Door Co., Inc., Lake Charles, La.

Davis Hardwood Co., San Francisco, Calif.

Davis Manufacturing Co., New Orleans, La.

Dealers Wholesale Supply, Inc., Detroit, Mich.

Deats Sash \& 2 Door Co., Los Angeles, Calif.

Deer Park Lumber Co., Deer Park. Wash.

DeJarnette, Charles Wagner, Des Moines, Iowa.

DeLancy Iumber Co., Lancaster, Ohio.

Deming \& Thompson Co.. Ine., Frankfort, Ind.

Dibble Lumber Co., The S. B., North Adams, Mass.

District of Columbia, Government of, Office of the

Municipal Architect, Washington, D. C.

Dixie Millwork Co., Ine., Hagerstown Md.

Donlin-Johnson Co., St. Ċloud, Minn.

East Point Lumber Co., East Point, Ga.

Elmer \& Moody Co., Seattle, Wash.

Empire Millwork Corporation, Corona, N. Y

English, Harold T., Hutchinson, Kans.

Epstein Co., N. B., Scranton, Pa.

Equity Lumber Co., The, Painesville, Ohio.

Estes Lumber Co., Birmingham, Ala.

Evans-MacArthur Co., New York, N. Y.

Evansville Sash \& Door Co., Inc., Evansville, Ind.

Everett \& Associates, H. F., Allentown, $\mathrm{Pa}$.

Farley \& Loetscher Manufacturing Co., Dubuque, Iowa.

Feldman-Wood-Products Co., Long Island City, N. Y

Fish \& Hunter Co., The, Rapid City, S. Dak.

Flannagan, Eric G., Henderson, N. C.

Flint Sash \& Door Co., Inc., Flint, Mich.

Florida, University of, School of Forestry, Gainesville, Fla.

Foltz \& Son, Herbcrt, Winter Haven, Fla.

Ft. Wayne Builders Supply Co., Ft. Wayne, Ind.

Foster \& Co., Inc., James P., Baltimore, Md.

Foster Lumber Co., R. S., Indianapnlis. Ind.

Frank \& Co., R. W., Salt Lake City, Utah.

Fuller, Robert K., Denver, Colo.

Fuller \& Co., W. P., Boise, Idaho., Portland, Oreg. and Seattle, Wash.

Furer, William C., Honolulu, Hawaii.

Galliher \& Huguely, Inc., Washington, D. C.

General Millwork Corporation, Utica, N. Y.

Gibson Door Co., The, Utica, N. Y.

Ginsberg \& Sons, Inc., D., Corona, N. Y.

Glendale Sash \& Millwork Corporation, Brooklyn, N. Y.

Grand Rapids Sash \& Door Co., Grand Rapids, Mich.

Great Lakes Sash \& Door Co., The, Cleveland, Ohio. Grimm Planing Mill, Albert C., Evansville, Ind. Hager \& Cove Lumber Co., Lansing, Mich.

Hallack \& Howard Lumber Co., The, Denver, Colo.

Hannaford \& Sons, Samuel, Cincinnati, Ohio.

Haralson \& Mott, Fort Smith, Ark.

Harbor Plywood Corporation of California, San Francisco, Calif

Harbor Plywood Corporation (Chicago Division), Chicago, Ill.

Harbor Sales Co., Inc., The, Baltimore, Md., and Washington, D. C.

Harris Brothers Co., Chicago, Ill.

Hasness, Carlisle D., Harrisburg, Pa.

Hastings \& Co., Inc., A. W., Somerville, Mass.

Hawkins Lumber \& Warehouse Co., Boston, Mass.

Helfensteller, Hirsch \& Watson, St. Louis, Mo.

Henderlong Lumber Co., Inc., Crown Point, Ind.

Henshaw \& Ellwanger, Inc., Denver, Colo.

Hoener, P. John, St. Louis, Mo.

Holsman \& Holsman, Chicagn, Ill.

Houston Sash \& Door Co., Houston, Tex.

Huber-Lanctot Housewrecking Corporation, Buffalo, N. Y.

Hurd Millwork Corporation, Medford, Wis.

Huttig Manufacturing Co., Muscatine, Iowa.

Huttig Sash \& Door Co., St. Louis, Mo., Charlotte, N. C., \& other cities.

Ideal Co., Waco, Tex.

Independent Lumber Co., The, Grand Junction, Colo.
Indiana Lumber \& Manufacturing Co., South Bend Ind.

International Steel Co., Revolving Door Division, New York, N. Y.

Interstate Lumber Co., Missoula, Mont.

Interstate Sash \& Door Co., The, Canton, Ohio.

Iron City Sash \& Door Co., Pittsburgh, Pa.

Iron Mountain City Lumber Yard, Iron Mountain, Mich.

Ivey, Inc., Edwin J., Seattle, Wash.

Jacksonville Sash \& Door Co., Inc., Jacksonville, Fla.

Jefferson Wood Products Co., Jefferson, Wis.

Jones Hardwood Co., San Francisco, Calif.

Jordan Millwork Co., Sioux Falls, S. Dak.

Keely Plywood Co., Hal, Pittsburgh, $\mathrm{Pa}$.

Keely \& Sons, S. S., Philadelphia, Pa.

Kelley, Frederic P., New York, N. Y.

Kellogg \& Sons Co., Charles C., Utica, N. Y.

Keystone Frame \& Manufacturing Co., Spokane, Wash.

Kilham, Hopkins, \& Greeley, Boston, Mass.

Kimball Lumber Co., Watertown, Mass.

Kimball \& Wilson, Inc., Detroit, Mich.

Kinzua Pine Mills Co., Kinzua, Oreg.

Kneeland Bigelow Distributing Co., Bay City, Mich. Kuebler Co., J. M., Wausau, Wis.

Kullberg Manufacturing Co, Minneapolis, Minn.

Kyle, Herbert S., Charleston, W. Va. (In principle.)

Lafayette Sash \& Door Factory, Lafayette, La.

Lank Woodwork Co., Inc., Washington, D. C.

Latenser \& Sons, John, Omaha, Nebr.

Law, Law, Potter \& Nystrom, Madison, Wis.

Lentz Co., A., Wauwatosa, Wis.

Leuckel \& Co., Inc.. A. K., Trenton, N. J.

Lewis Lumber Co., Spring Lake, N. J.

Lock City Manufacturing Co., Sault Ste. Marie, Mich.

Lockland Lumber Co., The, Lockland, Ohio.

Loeb, Laurence M., Elmsford, N. Y.

Loetscher \& Burch Manufacturing Co., Des Moines, Iowa.

Long Bell Lumber Co., The, Kansas City, Mo.

Lumber \& Millwork Co., of Philadelphia, The, Philadelphia, $\mathrm{Pa}$.

Lumbermen's Credit \& Warehouse Co., Kalamazoo, Mich.

Lumbermen's Door \& Trim Co., The, East Cleveland, Ohio.

Lumbermens Supply Co., Oklahoma City, Okla.

Lyman-Hawkins Lumber Co., The, Akron, Ohio.

Lyon-Gray Lumber Co., Dallas, Tex.

M \& M Wood Working Co., Portland, Oreg.

Mahoney Sash \& Door Co., The, Canton, Ohio.

Mansfield Lumber Co., Fort Smith, Ark.

Markland Contracting Co., M. B., Atlantic City, N. J.

Marquard Sash \& Door Manufacturing Co., The, Cleveland, Ohio.

Marquart Millwork Co., Oshkosh, Wis.

Martin, Egdar, Chicago, 111

Martin Lumber Co., Springfield, Mass.

Mason City Millwork Co., Mason City, Iowa.

Mason \& Co., George D., Detroit, Mich.

Mason \& Sons, Inc., A., Peru, N. Y.

Mauk Seattle Lumber Co., Seattle, Wash.

McCallum, D. D., Los Angeles, Calif.

McClelland Co., The, Davenport, Iowa.

McCoy \& Co., Inc., Lawrence R., Worcester, Mass. McGrath, R. A., Millwork Sales, Detroit, Mich.

McPhillips Manufacturing Co., Mobile, Ala.

Melander Co., C. T., East Orange, N. J.

Melrose Mill Co., Chicago, Ill.

Memphis Sash \& Door Co., Memphis, Tenn.

Merritt Lumber Yards, Inc., Reading, $\mathrm{Pa}$.

Miller Building Service, L. R., Grand Rapids, Mich.

Miller Manufacturing Co., Inc., Richmond, Va.

Miller \& Yeager, Terre Haute, Ind.

Missoula White Pine Sash Co., Missoula, Mont.

Montgomery \& Patteson, Charleston, W. Va.

Moore \& Co., Dallas, Tex.

Moore \& Galloway Lumber Co., Fond du Lac, Wis.

Mooser, William, San Francisco, Calif.

Morgan Co., Oshkosh, Wis.

Morgan Millwork Co., Baltimore, Md.

Morgan Sash \& Door Co., Chicago, Ill., and Oklahoma City, Okla.

Morns Plains Lumber \& Coal Co., The, Morris Plains, N. J.

Morrison-Merrill \& Co., Salt Lake City, Utah. 
Mueller, Hair \& Hetterich, Familton, Ohio.

Muhlenberg Brothers, Reading, Pa. (In principle.) Mummerlyn Lumber Co., H. J., Bennellsville, S. C. Mutual Millwork Co., Orlando, Fla.

Nashville Sash \& Door Co., Nashville, Tenn.

National Plywood Co., Inc., New York, N. Y.

National Manufacturing Co., Sterline, Ill.

Neal-Blun Co., Savannah, Ga.

Neal Millwork \& Supply Co., Oklahoma City, Okla.

Neumann \& Sons, William, Jersey City, N. J.

Newton Lumber \& Manufacturing Co., The, Colorado Springs, Colo.

Nicolai Door Manufacturing Co., Chicago, Ill.

Nicolai Door Sales Co., San Francisco, Calif.

Nielsen Construction Co., Harrisonburg, Va.

Noelke Lyon Manufacturing Co., Burlington, Iowa.

Northern Sash \& Door Co., Hawkins, W is.

Northside Building Supply Co., Doraville, Ga.

Nurenburg, W. S., Fort Worth, Tex.

O \& N Lumber Co., Menomonie, Wis.

Oettinger Lumber Co., Greensboro, N. C.

Officer, Gwynn, Berkeley, Calif.

Ohio City Sash \& Door Co., Dayton, Ohio.

Pacific Manufacturing Co., Santa Clara, Calif.

Pacific Mutual Door Co., Chicago, Ill. and St. Paul, Minn.

Paducah Sash \& Door Co., Paducah, Ky.

Parker Building Specialties, Inc., San Francisco, Calif.

Patten-Blinn Lumber Co., Los Angeles, Calif. (In principle.)

Pease Woodwork Co., Inc., Cincinnati, Ohio.

Peek \& Sons, S. H. East Aurora, Erie County, N. Y.

Pennsylvania, Commonwealth of, Department of Property \& Supplies, Harrisburg, $\mathrm{Pa}$.

Pepper, George W., Jr., Philadelphia, Pa.

Perlin Lumber Co., Brooklyn. N. Y.

Platt \& Brother, F. P., New York, N. Y

Porter-Haddey Co., Grand Rapids, Mich.

Porter Screen Co., Burlington, $\mathrm{Vt}$.

Queen City Sash \& Door Co., The, Cincinnati, Ohio. Quigley Co., J. R., Gloucester City, N. J

Radford Co., The, Oskosh, Wis., and Duluth, Minn.

Radford \& Sanders Inc., Baltimore, Md.

Ramsey \& Sons, Inc., A. H., Miami, Fla.

Rather, J. T., Jr., Houston, Tex.

Red River Lumber Co., The, Los Angeles, Calif., and Chicago, Ill.

Reeb Millwork Corporation, Roselle, N. J.

Resnikoff, Abraham, New York, N. Y.

Rinehimer Brothers Manufacturing Co., Elgin, Ill.

Roach \& Musser Co., Muscatine, Iowa.

Roberson \& Son, Inc., A., Binghamton, N. Y.

Rohert \& Co., Inc., A tlanta, Ga.

Roberts Corporation, U. N., Davenport, Iowa.

Rock Island Lumber Co., Cleveland, Ohio.

Rock Island Sash \& Door Works, Rock Island, Ill

Rockwell Brothers \& Co., Houston, Tex.

Roddis Lumber \& Veneer $\mathrm{Co}$, . Milwaukee, $\mathrm{W}$ is.

Rogers Lumber- Co., The T. H., Oklahoma City, Okla.

Rohrer Lumber Co., D. J., Clintonville, $W$ is.

Rounds \& Porter Co., Wichita, Kans.

Rudinger, Inc., C. R., South Kearny, N. J

Ruggles Lumber Co., Carlos, Springfield, Mass.

Russell \& Erwin Manufacturing Co., New Britain, Conn.

Rust Sash \& Door Co., Kansas City, Mo.

St. Louis Sash \& Door Works, St. Louis, Mo. Sanders Brothers Manufacturing Co., Ottawa,Ill. Sash, Door \& Giass Corporation, Richmond, Va. Scherer \& Co., Inc., Wm. C., Baltimore, Md. Schulzke, William H., Moline, Ill.

Scott Graff Co., Duluth, Minn.

Sears, Roebuck \& Co., Chicago, Ill.

Segelke \& Kohlhaus Co., La Crosse, Wis.

Semling Menke Co., Merrill, $W$ is.

Seneca Lumber \& Millwork Co., The, Fostoria, Ohio.

Seville Lumber \& Supply Co., Seville, Ohio.

Shannon Sash \& Door Co., Inc., Kansas City, Kans. Shenk Co., Henry, Erie, Pa.

Silbernagel \& Sons Co., Geo., Wausau, $W$ is.

Simons Inc., Minneapolis, Minn.

Sloan Lumber Co., Fort Worth, Tex.

Smith Co., Allen A., Toledo, Ohio.
Snell Sash \& Door Co., St. Paul, Minn.

Sothman Co., The Grand Island Nebr.

Southern Counties Gas Co. of California, Los Angeles, Calif.

Southwestern Sash \& Door Co., Joplin, Mo., Albu-

querque, N. Mex., and El Paso, Tex.

Spokane Pine Products Co., Spokane, Wash.

Spokane Sash \& Door Co., Spokane, Wash.

Spokane Woodworking Co., Spokane, Wash.

Standard Lumber \& Supply Co., Fort Wayne, Ind

Standard Manufacturing Co., The, Appleton, Wis.

Stark \& Co., Kansas City, Mo.

Steves Sash \& Door Co., San Antonio, Tex.

Stoetzel, Ralph E., Chicago, Ill.

Stokes \& Allyn, Portland, Oreg.

Sturtevant Millwork \& Lumber Corporation, Hicks-

ville, Long Island, N. Y.

Swan Lake Moulding Co., Klamath Falls, Oreg.

Swenson Construction Co., Kansas City, Mo.

Taylor Sash \& Door Co., Pensacola, Fla.

Teachout Sash, Door \& Glass Co., The, Dearborn, Mich.

Theiling-Lothman Manufacturing Co., St. Louis, Mo.

Thomas, Arthur E., Dallas, Tex.

Thorne, Henry Calder, Ithaca, N. Y

Throop-Martin Co., The, Columbus, Ohio.

Toombs \& Co., Springfield, Mo.

Trebing Manufacturıng Co., The, Cleveland, Ohio.

Trexler Lumber Co., Allentown, Pa.

Tulsa Rig, Reel \& Manufacturing Co, Tulsa, Okla.

Turner Lumber Co., J. C., Irvington, N. Y.

Underwood Coal \& Supply Co., Mobile, Ala.

Union Planing Mill, Stockton, Calif.

United Sash \& Door Co., Wichita, Kans.

Vallamont Planng Mill Co., Williamsport, Pa.

Vaughan \& Sons, Geo. C., Houston, Tex., and San Antonio, Tex.

Velde Lumber Co., Pekin, Ill.

Vetter Manufacturing Co., Stevens Point, Wis.

Victoria Sash \& Door Co., Inc., Shreveport, La.

Villaume Box \& Lumber Co., The, Saint Paul, Minn.

Virginia Poly technic Institute, Blacksburg, Va.

Vogel, Willis A., Toledo, Ohio.

Wabash Screen Door Co., The, Chicago, IIl.

Wahlfeld Manufacturing Co., Peoria, Ill.

Wanke Panel Co., Portland, Oreg.

W ashington Wood Working Co., Inc., Washington, D. C.

Watertown Sash \& Door Co., Watertown, S. Dak.

Weimer \& Sons, George, St. Albans, W. Va.

Welch, Carroll E., Huntington, N. Y.

West, Albert E., Bosten, Mass.

Western Door \& Plywood Corporation, Portland, Oreg.

Western Pine Manufacturing Co., Ltd., Spokane, Wash.

Wheelock, Inc., E. U., Los Angeles, Calif.

Whissel Lumber Co., Inc., L. N., Buffalo, N. Y.

White Pine Sash Co. of Illinois, Chicago, Ill.

Whitmer-Jackson Co., Inc., The, Buffalo, N. Y., Cleveland, Ohio, and Rochester, N. Y.

Whitmer Mills, Albuquerque, N. Mex.

Whittier Lumber \& Millwork Co., Newark, N. J.

Wholesale Building Supply, Inc., Oakland, Calif.

Wholesale Sash, Doors \& Millwork, Toledo, Ohio.

Wilkinson Co., Inc., The, Indianapolis, Ind.

Williams \& Hunting Co., Cedar Rapids, lowa.

Wimberly \& Thomas Hardware Co., Inc., Birming. ham, Ala.

Wolverine Shingle \& Lumber Co., Detroit, Mich.

Wood Glass Co., Syracuse, N. Y.

Wood Products Magazine, Chicago, Ill. (In principle.)

Young, Edwin, Wilmington, Del.

Zimmerman, A. C., Pasadena, Calif.

\section{U. S. GOVERNMEN'T}

Interior, Department of the, Office of Indian Affairs, Chicago, Ill.

Justice, Department of, Bureau of Prisons, Construction Division, Washington, D. C.

Veterans Administration, Washington, D. C.

War Department, W ashington, D. C.

War Production Board, Office of Civilian Require ments, Washington, D. C. (In principle.) 
